\title{
Inference and Analysis of Population Structure Using Genetic Data and Network Theory
}

\author{
Gili Greenbaum, ${ }^{*,+, 1}$ Alan R. Templeton, ${ }^{\neq, \S}$ and Shirli Bar-David ${ }^{\dagger}$ \\ *Department of Solar Energy and Environmental Physics and ${ }^{\dagger}$ Mitrani Department of Desert Ecology, Blaustein Institutes for Desert \\ Research, Ben-Gurion University of the Negev, 84990 Midreshet Ben-Gurion, Israel, ”Department of Biology, Washington \\ University, St. Louis, Missouri 63130, and §Department of Evolutionary and Environmental Ecology, University of Haifa, 31905 \\ Haifa, Israel
}

\begin{abstract}
Clustering individuals to subpopulations based on genetic data has become commonplace in many genetic studies. Inference about population structure is most often done by applying model-based approaches, aided by visualization using distancebased approaches such as multidimensional scaling. While existing distance-based approaches suffer from a lack of statistical rigor, model-based approaches entail assumptions of prior conditions such as that the subpopulations are at Hardy-Weinberg equilibria. Here we present a distance-based approach for inference about population structure using genetic data by defining population structure using network theory terminology and methods. A network is constructed from a pairwise genetic-similarity matrix of all sampled individuals. The community partition, a partition of a network to dense subgraphs, is equated with population structure, a partition of the population to genetically related groups. Community-detection algorithms are used to partition the network into communities, interpreted as a partition of the population to subpopulations. The statistical significance of the structure can be estimated by using permutation tests to evaluate the significance of the partition's modularity, a network theory measure indicating the quality of community partitions. To further characterize population structure, a new measure of the strength of association (SA) for an individual to its assigned community is presented. The strength of association distribution (SAD) of the communities is analyzed to provide additional population structure characteristics, such as the relative amount of gene flow experienced by the different subpopulations and identification of hybrid individuals. Human genetic data and simulations are used to demonstrate the applicability of the analyses. The approach presented here provides a novel, computationally efficient model-free method for inference about population structure that does not entail assumption of prior conditions. The method is implemented in the software NetStruct (available at https:// giligreenbaum.wordpress.com/software/).
\end{abstract}

KEYWORDS community detection; hierarchical population structure; modularity; subpopulations

NFERENCE of population structure from genetic data is often used to understand underlying evolutionary and demographic processes experienced by populations, and is an important aspect of many genetic studies. Such inference is mainly done by clustering individuals into groups, often referred to as demes or subpopulations. Evaluation of population structure and gene flow levels between subpopulations allows inference about migration patterns and their genetic

Copyright (C) 2016 by the Genetics Society of America doi: 10.1534/genetics.115.182626

Manuscript received September 7, 2015; accepted for publication February 3, 2016; published Early Online February 16, 2016.

Supplemental material is available online at www.genetics.org/lookup/suppl/doi:10. 1534/genetics.115.182626/-/DC1.

${ }^{1}$ Corresponding author: Mitrani Department of Desert Ecology, Blaustein Institutes for

Desert Research, Ben-Gurion University of the Negev, Sede Boqer Campus, 84990

Midreshet Ben-Gurion, Israel. E-mail: gili.greenbaum@gmail.com consequences (Templeton 2006; Allendorf et al. 2012). As sequencing of larger portions of the genome is becoming more readily available, there is an increasing need for a variety of computationally efficient statistically testable methods for such inference.

Analysis of population structure can be done at the subpopulation-population level by assuming putative subpopulations and studying how these relate genetically [e.g., F-statistics, analysis of molecular variance (AMOVA) (Excoffier et al. 1992), phylogenetic methods (Cavalli-Sforza and Edwards 1967; Saitou and Nei 1987; Pickrell and Pritchard 2012)], or at the individual subpopulation level by attempting to cluster individuals to subpopulations. The methods for clustering individuals based on genetic data can be further divided into two categories: model-based approaches and distance-based approaches (Pritchard et al. 
2000; Alexander et al. 2009; Wollstein and Lao 2015). Model-based approaches evaluate the likelihood of the observed data, assuming that they are randomly drawn from a predefined model of the population, e.g., that there are $K$ subpopulations and that these subpopulations are at Hardy-Weinberg equilibrium (HWE). Distance-based approaches aim at identification of clusters by analysis of matrices describing genetic distances or genetic similarities between individuals or populations, e.g., by visualization using multidimensional scaling (MDS) methods such as principle components analysis (PCA). Distance-based methods are usually model-free and do not require prior assumptions, as with the model-based methods. Over the last decade or so, model-based methods have been more dominant as procedures for inference about population structure, mostly with implementation of Bayesian clustering and maximumlikelihood techniques in programs such as STRUCTURE, ADMIXTURE (Alexander et al. 2009), and BAPS (Corander et al. 2003). It has been pointed out that distance-based methods have several disadvantages (Pritchard et al. 2000): they are not rigorous enough and rely on graphical visualization, they depend on the distance measure used, it is difficult to assess the significance of the resulting clustering, and it is difficult to incorporate additional information such as geographic location of the samples. Jombart et al. (2008), and Yang et al. (2012) address this last concern. Given these disadvantages, it would seem that distance-based measures are less suitable for statistical inference about population structure. However, model-based approaches suffer from the need to restrict interpretation of the results by heavily relying on the prior assumptions of the model, e.g., that the populations meet certain equilibria conditions such as migration-drift or HWE (Pritchard et al. 2000).

There has recently been a flourish of network theory applications to genetic questions in genomics (Forst 2002), landscape genetics (Garroway et al. 2008), and population structure at the subpopulation-population level (Dyer and Nason 2004; Rozenfeld et al. 2008; Ball et al. 2010; Munwes et al. 2010). Recently, a network-based visualization tool, NETVIEW (Neuditschko et al. 2012), of fine-scale genetic population structure, using a superparamagnetic clustering algorithm (Blatt et al. 1996), has been proposed and applied successfully to analysis of livestock breeds (Burren et al. 2014; Neuditschko et al. 2014), and other network clustering approaches have also been implemented on genetic data (Cohen et al. 2013). However, these methods still suffer from the many disadvantages of distance-based clustering approaches, and a more rigorous and statistically testable distance-based approach is still missing.

Development of a suitable distance-based network approach that will coherently address inference about population structure from genetic data, and not suffer from the disadvantages listed earlier, necessitates a clear definition of genetic population structure in equivalent network theory terminology. A genetically defined subpopulation is commonly thought of as a group of individuals within a population who are more genetically related (or more genetically similar) to each other than they are to individuals outside the subpopulation, as a result of many possible genetic processes such as genetic drift, migration, mutation, and selection. In a network, a group of nodes that are more densely and strongly connected within the group than outside the group relative to the given topology of the network is called a community (Newman and Girvan 2004). Therefore, in network theory terminology, the equivalent of a genetic population structure should be the community partition of a network constructed with individuals as nodes and edges defined using an appropriate genetic distance or similarity measure. In network science, clustering nodes into groups has been studied extensively, and specifically community detection has attracted much interest (Girvan and Newman 2002). Because there is no single rigid definition of a community, and because identifying optimal partitions is computationally expensive, many approaches and algorithms to optimally detect communities in networks have been proposed (Lancichinetti and Fortunato 2009; Fortunato 2010).

We propose a network-based approach for analyzing population structure based on genetic data. We show that by applying recent advances in network theory, it is possible to design a distance-based approach that overcomes the limitations of other distance-based approaches, and does not suffer from the disadvantages of model-based approaches. We also show how rigorous statistical inference can be incorporated into this network-based approach in a manner that does not entail prior assumptions or conditions about the data.

The process can be used with a large number of loci (e.g., microsattelites or SNPs) because it is computationally efficient in regard to the number of loci incorporated in the analysis. Moreover, we define a new measure for the strength to which an individual is associated with its assigned community, called strength of association (SA), and we show how strength of association distribution (SAD) analysis can be used to infer further details regarding population structure, such as gene flow patterns of each subpopulation and identification of potentially hybrid individuals. The analysis is demonstrated on genetic data from the human population extracted from the HapMap Project (International HapMap Consortium 2003), as well as on simulated data. In addition to presentation of a new distance-based method for population structure inference, we believe that defining the problem of genetic population structure analysis in network terminology will allow future adoption and adaptation of network methods and techniques to address population genetic questions.

\section{Methods}

In this section we provide the relevant theory and describe a network-based approach for constructing genetic networks and inferring population structure by detecting community 
partitions in these networks. Following detection of community structure, we propose an additional exploratory analysis, based on a measure of the strength of association of individuals to communities, that may shed light on finer details of the community structure and therefore on the population structure.

\section{Constructing networks from genetic data}

A network is a set of discrete entities, nodes, where each pair of nodes may be connected by an edge, possibly characterized by a weight. Networks can be described by adjacency matrices, where the element in column $i$ and row $j$ is the weight of the edge connecting node $i$ and node $j$. For most network applications, dyads connected by edges with high-valued weights are interpreted as being strongly connected. Therefore, a genetic-similarity matrix (a matrix describing some measure of genetic similarity or relatedness between all pairs of individuals, based on their genotypes) of a population also can be regarded as the adjacency matrix of a genetic-similarity network. The more classic genetic-distance (genetic dissimilarity) matrices are similar, except that in these, high values indicate weakly connected dyads, and therefore, such matrices must be appropriately transformed to be considered network adjacency matrices. There is no fundamental difference, for our purpose, between similarity and distance matrices. Many genetic similarity, distance, and relatedness measures have been proposed (Oliehoek et al. 2006), but if we restrict the discussion to symmetric similarity measures, where similarity between individual $i$ and $j$ is the same as between individual $j$ and $i$, the genetic network thus described is a weighted undirected network, in which each edge is characterized by a weight but does not have directionality. Because we would like to consider not only allele sharing between individuals but also differences in allele frequencies between subpopulations, we further restrict the discussion to genetic similarity measures that are expressed relative to allele frequencies in a reference population, i.e., measures that incorporate the allele frequencies of the total sampled population. These measures should not incorporate allele frequencies other than those of the total sample (e.g., allele frequencies in sample sites or other locally defined sampled populations) because this would mean that the null hypothesis is other than that there is no population structure. From a distance-matrix perspective, the null hypothesis is that the adjacency matrix, appropriately transformed, is a symmetric individual-pairwise distance matrix such that all individuals are equally distant from the multilocus centroid of the total population with no subclustering.

In a network such as that just described, the strength of the connection between each dyad is relative to the genetic similarity between them, where shared rare alleles convey a stronger connection than do common alleles. This is different from many commonly used distance measures for construction of individual-level distance matrices, such as in
AMOVA (Excoffier et al. 1992), where distances are measured by mismatch of alleles, regardless of their frequencies. Because even unrelated individuals may share many alleles, especially when many loci are examined, it is likely that this network will be extremely dense. It therefore may be useful, both from a computational point of view and in order to emphasize strong genetic relations within the population so as to increase the detection power of network procedures, to remove edges that describe weak connections. This can be done in different ways, but the most straightforward approach is to remove edges with weights below a certain threshold, which is the approach we implement here. In this way, a sparser network that consists of strong relatedness interconnections is attained.

Because using different thresholds will result in different networks that may give, for the analyses that follow, different population structures, we recommend systematically exploring different threshold values. For very low threshold values, many weak relatedness interconnections will be included in the network, which may result in very dense networks that could mask related groups within the population. Very high thresholds may result in the network breaking down into many disconnected components (a network component is a group of nodes that are connected within themselves but are not connected to any other node in the network), up to a point when the network includes only very small groups of connected nodes. Such networks are most likely not informative of population structure because they represented too few related dyads, and the community partition likely will consist of many one- or two-node communities. Each community is confined to be within a component, and if the network consists of many small components, then the community partition is constrained to include many small communities. Therefore, the informative structures should be detected at the intermediate thresholds, and different thresholds in this range may describe structure at different hierarchical levels (see Analysis of human SNP data for an example of a systematic exploration of threshold values).

\section{Network communities and genetic population structure}

In network theory, the term community refers to a subset of nodes in a network that are more densely connected to each other than to nodes outside the subset (Newman 2006). There are now several algorithms for efficiently partitioning a network into communities (Lancichinetti and Fortunato 2009; Fortunato 2010). Most commonly, a partition of a network into communities is evaluated by calculating the modularity of the partition, a quality measure (between -1 and 1) indicating whether the partition is more or less modular than would be expected if connections were randomly distributed (Newman 2004b). The modularity of a particular community partition of a weighted network $A$ (a network with weights assigned to its edges) is defined as the weight of the intracommunity connections minus the expected weight of the intracommunity connections in a random 
network preserving the edge weights of each node (Newman 2004a):

$$
Q=\frac{1}{A^{*}} \sum_{\mathrm{i}, \mathrm{j}}\left(A_{\mathrm{ij}}-\frac{1}{A^{*}} \sum_{\mathrm{k}} A_{\mathrm{ik}} \sum_{1} A_{\mathrm{lj}}\right) \delta\left(c_{\mathrm{i}}, c_{\mathrm{j}}\right)
$$

where $A^{*}=\sum_{\mathrm{k}, 1} A_{\mathrm{lk}}$ is the sum over all edge weights in the network, and $\delta\left(c_{\mathrm{i}}, c_{\mathrm{j}}\right)$ is a delta function with value 1 if nodes $i$ and $j$ are in the same community and 0 otherwise. A positive modularity value indicates that the partition is more modular than expected. Many community-detection algorithms try to approximate an optimal partition, in which the modularity measure is maximal over all possible partitions. A partition consisting of one community, which includes all nodes in the network, results in a modularity of zero, and therefore, for every network, the optimal partition, maximizing the modularity, is always nonnegative. Community-detection algorithms do not generally require a priori knowledge of the number of clusters present in the network.

Because in a genetically subdivided population the individuals in a subpopulation are expected to be more highly related in comparison to a random redistribution of relatedness levels between individuals, communities in the genetic network are expected to coincide with the subpopulations of the underlying population structure. We therefore propose that population structure can be ascertained by constructing a genetic network based on a genetic similarity measure and then applying community-detection methods to identify a partition that maximizes modularity. Because it is possible that an optimal partition consists of just one community (i.e., the entire network), community-detection algorithms also can identify scenarios with no population subdivision.

A partition of the network into two or more communities may be indicative of population structure, but community partitions also may be detected in panmictic populations owing to chance alone. In order to test whether the detected population structure is significant, we test whether it is significantly different from the null hypothesis of no population structure. Several approaches have been suggested to evaluate the statistical significance of community partitions (Fortunato 2010), but here we pursue a significance test based on permutations of the genetic network that focuses on testing whether the null hypothesis can be rejected or not. With no population structure, dense subgraphs are not expected to appear in networks constructed as described earlier, and if such subgraphs do appear, the modularity of an optimal partition of the network should be relatively low. In randomly permuted networks, dense subgraphs are expected to be present as often as in unstructured populations, and hence, optimal partitions of permuted networks can be used to represent the expected modularity of optimal partitions of unstructured populations. If the modularity of the detected community partition is not significantly higher than modularities of optimal partitions of the permuted networks, we cannot reject the null hypothesis. We can therefore use permutations to test the statistical significance of detected community partitions.

With application of this significance test, population genotypes are considered to describe a structured population if, and only if, more than one community is detected, and the detected partition is more modular than would be expected in a population with no structuring. Preferably, the permutation should be done at the level of the genotypes because this will ensure that all structural properties of the the permuted network, such as the degree distribution and global clustering coefficient (Opsahl and Panzarasa 2009), will be similar to those of the original network. However, permutations of the genotypes require constructing many matrices, which would be computationally expensive when many loci are involved. As an alternative, permutations can be done at the adjacency matrix level, with symmetry-preserving permutations of the matrix. Such permutations will preserve the main structural property of the network, the edge degrees, but not the global clustering coefficient. While, in practice, this should not affect the modularity of optimal partitions because the clustering coefficient measures clustering only at the immediate-neighbor level and not the modular structure of the network, permutation of genotypes should be used whenever feasible.

\section{Strength of association distribution (SAD) analysis}

Revealing the division of the population into subpopulations may shed light on many aspects of the underlying evolutionary and ecological processes, but more information can be attained by further analyzing the characteristics of the partition. The partitioning of the network into dense subgraphs, as presented earlier, does not convey information regarding how important each individual is to the detected partition. Here we introduce a measure intended to evaluate this aspect, the strength of association (SA) of individual $i$ to its community. Given a community partition $C$ and an individual $i$, we define the strength of association $S A$ as

$$
S A(C, i)=\min _{\substack{\mathrm{k} \\ \mathrm{C}_{\mathrm{k}}(\mathrm{i}) \neq \mathrm{C}}}\left(Q_{\mathrm{C}}-Q_{\mathrm{C}_{\mathrm{k}}(\mathrm{i})}\right)
$$

where $Q_{\mathrm{C}}$ is the modularity of the partition $C$, and $C_{\mathrm{k}}(i)$ is the partition identical to $C$ except that node $i$ is assigned to community $k$ instead of its original community. Thus high $S A$ values indicate that the partition $C$ is sensitive to the assignment of $i$ and that the assignment of $i$ to its community is essential, whereas low $S A$ values indicate that there is another community that the individual is well assigned to. From a population genetics perspective, the measure evaluates how strongly individuals are related to the group to which they were assigned, and $S A$ is expected to be low when individuals are recent descendants from individuals from more than one subpopulation. For example, potential hybrids are expected to show low $S A$ values, and the $k$ that minimizes 


\section{A}

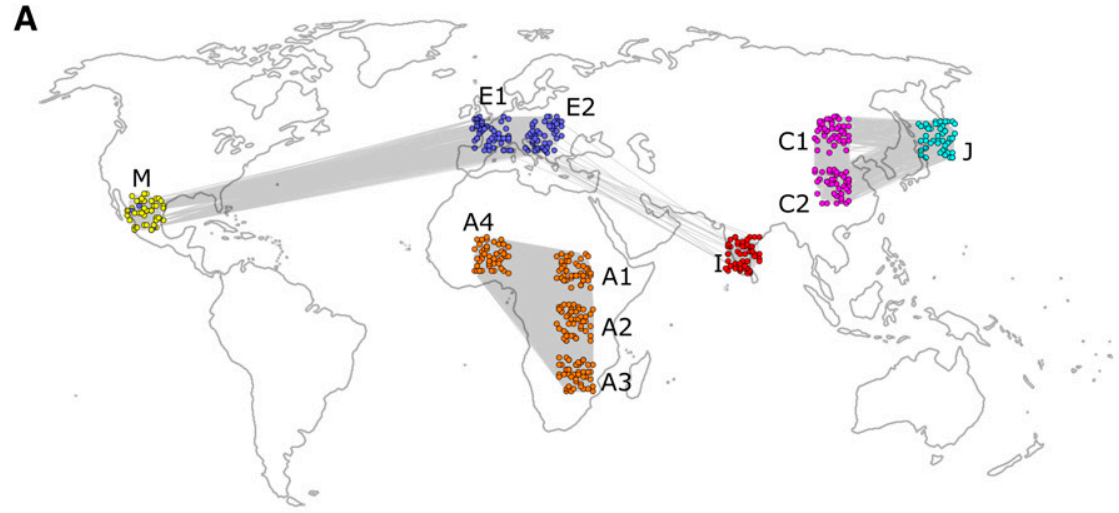

B
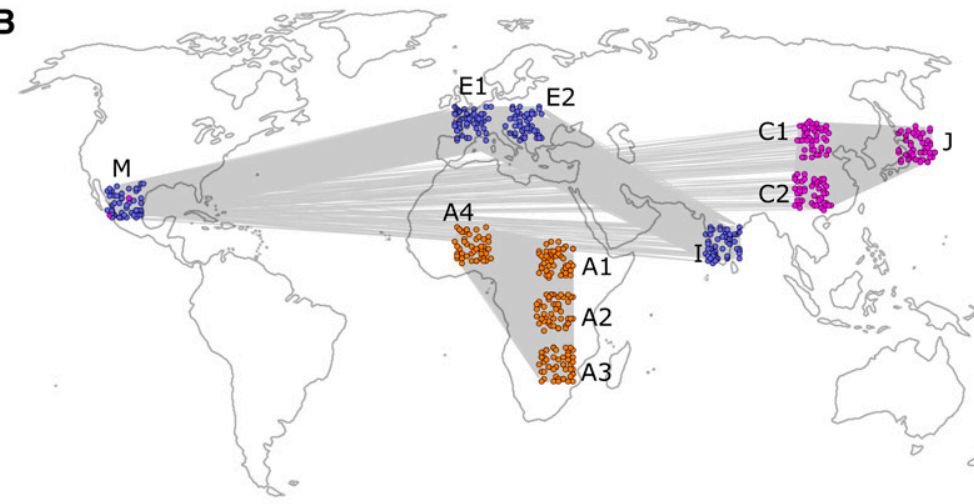

C

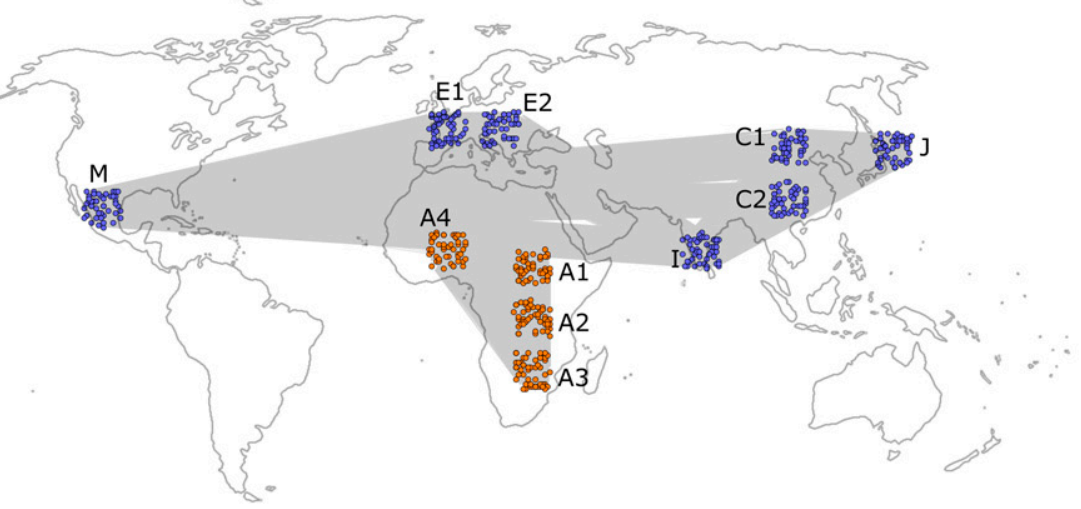

A1 - African ancestry Americans

A2 - African, Kenya

A3 - African, Masai

A4 - African, Nigeria

E1 - European

E2 - European ancestry Americans

C1 - Han Chinese

C2 - Chinese ancestry Americans

J - Japanese

I - Indian ancestry Americans

M - Mexican ancestry Americans

Figure 1 Community detection on three networks with different thresholds. Each node represents an individual, with colors representing the community assigned by the community-detection algorithm. (A) High threshold (0.207 for East Asian component, 0.198 for the rest of the network). (B) Medium threshold (0.194). (C) Low threshold (0.188). For visualization purposes, individuals are placed on the world map roughly corresponding to their ancestry.

Equation 2 is the probable origin of the second lineage of the individual (see Identifying hybrids and recently admixed individuals for an example).

The $S A$ measure is a measure at the individual level, taking into account genetic data of the entire population. We introduce an exploratory analysis that evaluates characteristics of subpopulations relating to the cohesion of the subpopulation and the association of individuals with the subpopulation, the strength of association distribution (SAD) analysis. This analysis examines the distribution of the $S A$ values of the different communities and compares the statistical attributes of these distribution (e.g., the mean, variance, and skew of the $S A$ distributions). Because different scenarios are expected to result in different cohesions of the subpopulations, it may be possible to hypothesize what underlying processes were responsible for shaping the genetics of the population.

For example, a closed, disconnected subpopulation is expected to display a narrow SAD with a high mean (high community cohesion) because, in a closed population, individuals will be strongly related relative to the entire population, and individuals descended from lineages outside the subpopulation are rare. A subpopulation experiencing constant moderate gene flow levels is expected to display a wide or left-skewed SAD with a high mean because there should be many individuals with lineages that are mostly from the subpopulation, but recent migrants and descendants of recent migrants are expected to have low $S A$ values, increasing the variance and the left-skewness of the distribution. 


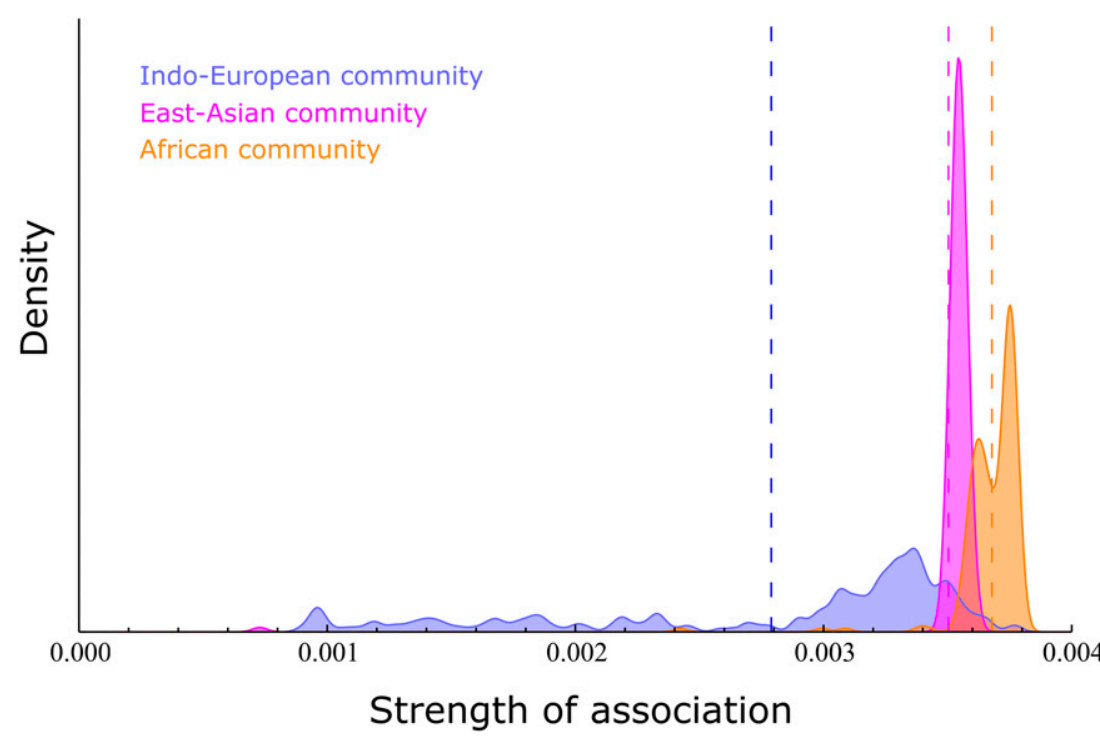

Figure 2 Strength of association distribution (SAD) analysis for the network in Figure 1B. Shown are the distributions of the $S A$ values for each of the three communities detected. Mean SA for each community is indicated by a dashed line.

A subpopulation experiencing constant strong gene flow levels is expected to display a SAD with a low mean because many individuals will be descendants of migrants.

\section{Analysis of human SNP data}

In order to demonstrate the applicability of the network approach to infer population structure and the SAD analysis, we have selected a set of human SNP data extracted from the HapMap database (International HapMap Consortium 2003). This data set is well suited for the demonstration of a new approach because it is taken from a population in which structure and demographic history are well known from archaeological, historical, and genetic studies. The genetic data for this analysis consisted of 50 randomly selected individuals from each of the 11 sampled populations of the HapMap Project (overall, 550 individuals): Africanancestry Americans $\left(A_{1}\right)$, Africans from West Kenya $\left(A_{2}\right)$, Masai Africans $\left(A_{3}\right)$, Africans from Nigeria $\left(A_{4}\right)$, Europeans from Italy $\left(E_{1}\right)$, European-ancestry Americans $\left(E_{2}\right)$, Han Chinese $\left(C_{1}\right)$, Chinese-ancestry Americans $\left(C_{2}\right)$, Japanese $(\mathrm{J})$, Indian-ancestry Americans (I), and Mexican-ancestry Americans (M). 1000 polymorphic SNPs from each autosome were randomly selected (overall 22,000 sites per individual). In order to compare the results with a modelbased approach, the same data were analyzed with the most widely used model-based software, STRUCTURE (Pritchard et al. 2000).

\section{Network construction}

A genetic network was constructed from the genotypes (without any information on the original grouping of the individuals) using, for calculation of genetic similarity, a simple symmetric frequency-weighted allele-sharing similarity measure. Analogous to the molecular similarity index (Li and Horvitz 1953; Oliehoek et al. 2006), we defined the frequency-weighted similarity at locus $l$ for diploid individual $i$ with alleles $a$ and $b$, with frequencies $f_{\mathrm{a}}$ and $f_{\mathrm{b}}$ (in the total sample), respectively, and individual $j$ with alleles $c$ and $d$ :

$$
S_{\mathrm{ij}, 1}=\frac{1}{4}\left[\left(1-f_{\mathrm{a}}\right)\left(I_{\mathrm{ac}}+I_{\mathrm{ad}}\right)+\left(1-f_{\mathrm{b}}\right)\left(I_{\mathrm{bc}}+I_{\mathrm{bd}}\right)\right]
$$

where $I_{\mathrm{ac}}$ is 1 if alleles $a$ and $c$ are identical and 0 otherwise, and the other indicators are similarly defined. Note that this measure is a multiallelic measure and is commutative with respect to $i$ and $j$. Given a sample with $L$ loci, the weight of the edge connecting individuals $i$ and $j$ is defined as the mean frequency-weighted similarity over all loci:

$$
A_{\mathrm{ij}}=\frac{1}{L} \sum_{\mathrm{l}=1}^{\mathrm{L}} S_{\mathrm{ij}, 1}
$$

The relatedness measure defined in Equation 3 is a very simple symmetric relatedness measure that measures diversity relative to the entire population, because it takes into account the allele frequencies at the level of the entire population (with sharing of rare alleles conveying a stronger connection than sharing of common ones). Other, more sophisticated, measures are likely to construct more accurate networks, and may be specific to the type of marker considered (e.g., for microsatellites, the length of the repeat might be taken into account) or include additional information (e.g., geographic locations of the samples). The formulation presented here is designed to analyze diploid populations, but it can be easily generalized to any level of ploidy.

\section{Community partition}

Currently, many algorithms are used for detecting population structure, relying on different network theory concepts [reviewed by Lancichinetti and Fortunato (2009) and Fortunato (2010)]. We have used several of the commonly employed algorithms, implemented using igraph (Csárdi and Nepusz 2006); they are presented in Supplemental Material, 
File S1, and we show here the results from the classic GirvanNewman algorithm (Girvan and Newman 2002). Results using different algorithms are not qualitatively different.

The edge-removal threshold parameter was explored systematically by examining thresholds from 0 until all edges were removed in steps of 0.001 . For very low thresholds (0-0.181), the constructed networks were very dense, and no population structure was detected (a single community, that included all nodes, was detected). As mentioned earlier, this is to be expected from all but the most distinctly structured populations because including connections between very weakly related individuals decreases the capability of the community-detection algorithms to detect dense subgraphs within the network. For very high thresholds $(>0.209)$, the networks break up into many disconnected components, many of which include only one or two nodes. Such networks cannot be coherently analyzed for communities (see Methods).

For thresholds between 0.182 and 0.208 , different community partitions were detected for different threshold ranges (Figure 1). For thresholds in the range 0.182-0.188, two communities were detected, and Figure 1C shows results for threshold 0.188, referred to as "low threshold". For the range 0.189-0.195, three communities were detected, and Figure 1B shows results for threshold 0.194, referred to as "medium threshold". For thresholds above 0.196, the network was no longer connected and broke into several components, most notably a dense East Asian component, and the rest of the network was composed of one or more components. For the range $0.196-0.200$, one community was detected in the East Asian component and four communities in the rest of the network. For thresholds above 0.201 , only the East Asian component remained intact, while the rest of the network broke into many small components and could no longer be meaningfully analyzed. The East Asian component consisted of one community for the threshold range 0.196 0.206 and two communities for the threshold range $0.207-$ 0.208 . Figure $1 \mathrm{~A}$ shows the results of the community partition with threshold 0.207 of the East Asian component, with two communities, and for threshold 0.198 for the rest of the network, with four communities (referred to as "high threshold"). Within the edge-removal threshold ranges mentioned earlier, there was no significant change in the assignment of the individuals to communities. Therefore, three qualitatively different community partitions of the network into communities have been found by systemically testing different edge-removal thresholds, with either two, three, or six communities for low, medium, and high thresholds, respectively (Figure 1).

Permutation tests using 1000 permutations of the genotypes were conducted, and all detected community partitions were strongly significant $(P \leq 0.001)$. With the low threshold, the partition corresponded to an African-non-African division (Figure 1C), with the medium threshold to an AfricanIndo-European-East Asian division (Figure 1B) and with the high threshold to one of six communities: African, Indian,

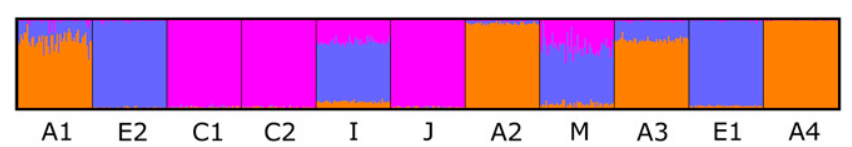

Figure 3 Model-based analysis of human SNP data assuming three subpopulations $(K=3)$ using the program STRUCTURE. The sampled population labels are the same as in Figure 1. The colors of the subpopulations correspond to the colors in Figure $1 \mathrm{~B}$ and Figure 2.

European, Mexican, Chinese, and Japanese (Figure 1A) (Some of the other community-detection algorithms also detected the Masai population as a distinct community for the high threshold; see File S1.) The trend where higher thresholds reveal more detailed structure is correlated with the historically known patterns of human population differentiation. The low-threshold coarse division of the population into two groups corresponds with the more ancient "out-ofAfrica" migrations, the medium-threshold division of the Eurasian population corresponds with the more recent migrations to Asia and the long-lasting Indo-European ethnic ties, and lastly, the high thresholds correspond with the most recent events of migration and isolation leading to the formation of cohesive populations in India, Japan, and Mexico.

The analysis with STRUCTURE was done for different $K$ values ( $K$ is the number of subpopulations assumed by the model). There is no statistical test available to evaluate the significance of the results for different models, but the most widely used heuristic is the one presented by Evanno et al. (2005). This heuristic shows that the most likely $K$ value is $K=2$, but $K=3$ and $K=6$ are also indicated as likely values (File S2, Figure A). For $K=2$ and $K=3$, the partition was the same as with the network approach (File S2, Figure B). For $K=6$, the detected partition consisted of five of the six subpopulations detected by the network approach: African, Indian, European, Mexican, and East Asian. The JapaneseChinese division was not detected, but the Masai individuals, assigned to the African subpopulations, also were shown to likely belong to a sixth subpopulation (File S2, Figure B).

\section{$S A D$ analysis}

As a demonstration of the SAD analysis, the network with medium threshold (Figure 1B) was analyzed, and the distribution of the $S A$ values for the three communities detected are shown in Figure 2. Figure 3 shows the equivalent analysis of the model-based results using STRUCTURE and assuming three subpopulations $(K=3)$.

The SAD of the East Asian community (Figure 2) has a high mean and is a very narrow distribution, consistent with a subpopulation experiencing limited gene flow. This can be explained by the known historical trend of the relative isolation of East Asia from Europe and Africa.

The SAD of the Indo-European community (Figure 2) is the one with the lowest mean $S A$ and is a wide, left-skewed distribution, consistent with a subpopulation with defined core and periphery that experienced extensive gene flow relative to the other subpopulations. Given that the individuals 


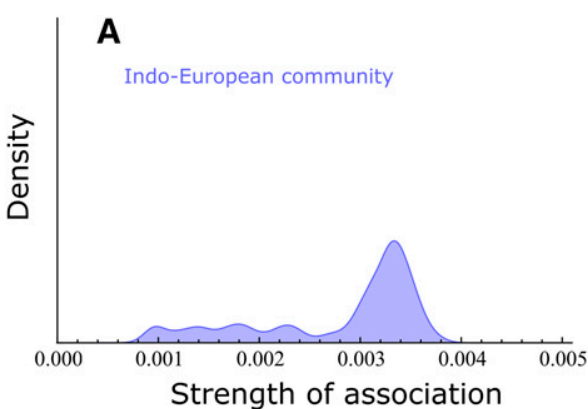

Strength of association

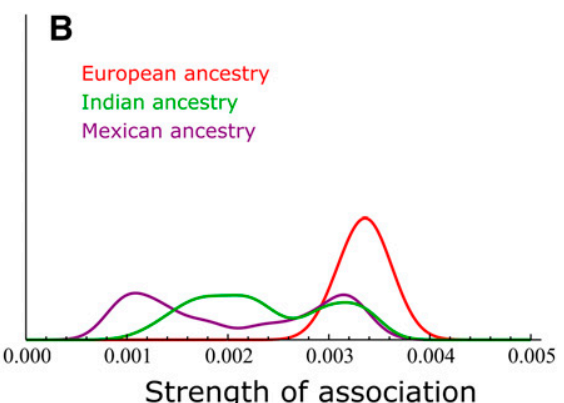

Figure 4 Strength of association distribution (SAD) analysis for the Indo-European (blue) community. (A) Distribution of the IndoEuropean community as shown in Figure 2. (B) SA distribution of the individuals in the community for different sampled subpopulations. It can be seen that the individuals with European ancestry are responsible for the higher $S A$ values in the distribution in $A$, while the individuals with Mexican or Indian ancestry have lower association with this community. belonging to this community are from European, Indian, or Mexican ancestry, a probable explanation is that the core consists of the two European sampled populations and that the Indian- and Mexican-ancestry individuals have lower association with this group. This can be clearly observed when the distribution is decomposed to three distributions based on the sampled populations (Figure 4).

The distribution of the African community (orange) has a high mean and is narrow and bimodal. This is consistent with a cohesive subpopulation with limited gene flow but also that two or more distinct subgroups exist within the population, with different levels of association with the community. Figure 5 shows the decomposition of the distribution to the four sampled populations composing it, and it can be seen that the the bimodality can be explained by the fact that the Masai population $\left(\mathrm{A}_{3}\right)$ is found to be a distinct population, as detected by some of the community-detection algorithms (File S1). The STRUCTURE analysis also points out to this possibility; as for $K=6$, it seems that the Masai individuals could possibility be assigned to a different subpopulation, although they are more likely to be assigned to the African subpopulation (File S2).

Comparing these results with the results from the modelbased analysis, both methods show that the African subpopulation is composed of the same group of individuals (Figure $1 \mathrm{~B}$ and Figure 3). With the model-based analysis, it can be seen that while individuals from Kenya $\left(\mathrm{A}_{2}\right)$ and Nigeria $\left(\mathrm{A}_{4}\right)$ have almost no probability to be assigned with other subpopulations, African-ancestry Americans $\left(\mathrm{A}_{1}\right)$ and Masai individuals $\left(A_{3}\right)$ have a nonnegligible probability of being assigned to other subpopulations, which could be interpreted as that these two groups, while belonging to the African subpopulation, have experienced more gene flow from other subpopulations, mostly from the Indo-European subpopulation. This finding is similar to that found with network analysis: these groups are likely to have experienced more gene flow, indicated by the lower mean $S A$ of both Masai and Africanancestry Americans than that of the Nigerian and Kenyan groups (Figure 5). However, the $S A$ distributions of $\mathrm{A}_{1}$ and $\mathrm{A}_{3}$ are quite different, which implies different evolutionary histories. The $A_{1} S A D$ is skewed with a long left tail, indicating that there are a number of African-ancestry Americans who are significantly less associated with the community and are possibly recently admixed individuals. The $\mathrm{A}_{3} \mathrm{SAD}$ has a low mean but is symmetric without a wide or skewed tail, possibly indicating that the Masai population has experienced more gene flow, but not in recent times. The recent admixture in the African-ancestry Americans group is consistent with recent higher gene flow experienced by the Africanancestry Americans from other American groups.

\section{Analysis of simulated data}

In order to get a better intuition on the performance of the method, and specifically on the SAD analysis and detection of hybrid and recently admixed individuals, several scenarios were simulated. These simulations consisted of three diploid populations $\left(\mathrm{P}_{1}, \mathrm{P}_{2}\right.$, and $\left.\mathrm{P}_{3}\right)$ of size $N$ ( $2 N$ haploids) that split $N$ generations ago from an ancestral population, also of size $N$. Following the split, migration is assumed to have occurred only between $\mathrm{P}_{1}$ and $\mathrm{P}_{2}$ at rate $m$ (proportion of the population per generation). This allowed us to explore the effects of gene flow between $\mathrm{P}_{1}$ and $\mathrm{P}_{2}$ in comparison with an isolated population, $\mathrm{P}_{3}$. Four scenarios with different levels of gene flow between $\mathrm{P}_{1}$ and $\mathrm{P}_{2}$ were examined: no gene flow $\left(S_{1}\right)$, low gene flow $\left(S_{2}\right)$, medium gene flow $\left(S_{3}\right)$, and high gene flow $\left(\mathrm{S}_{4}\right)$. Table 1 summarizes $m$ values for the different scenarios. The coalescence process for 5000 independent SNPs and for $N=50$ was simulated using the coalescence simulator SIMCOAL2 (Laval and Excoffier 2004).

\section{SAD analysis of simulated scenarios}

For the four simulated scenarios, genetic networks were constructed using Equation 4, as done for the human genetic data explained earlier. Community detection, using the computationally efficient Fast Greedy algorithm (Clauset et al. 2004), was performed for edge-removal thresholds starting from 0 in steps of 0.01 until the network was decomposed to many disconnected components. In scenarios $S_{1}$ and $S_{2}$, three communities were detected for all thresholds examined, fully corresponding with the three simulated populations (we therefore refer to these communities as $\mathrm{P}_{1}, \mathrm{P}_{2}$, and $\mathrm{P}_{3}$ ). In scenario $S_{3}$, two communities were detected for low thresholds (0-0.04), fully corresponding to the combined population of $\mathrm{P}_{1}$ and $\mathrm{P}_{2}$ and to population $\mathrm{P}_{3}$ (we refer to these as $\mathrm{P}_{1} \mathrm{P}_{2}$ and $\mathrm{P}_{3}$ ), while for high thresholds $(0.05)$, three communities were detected, fully corresponding to the three simulated populations. For scenario $\mathrm{S}_{4}$, two communities 

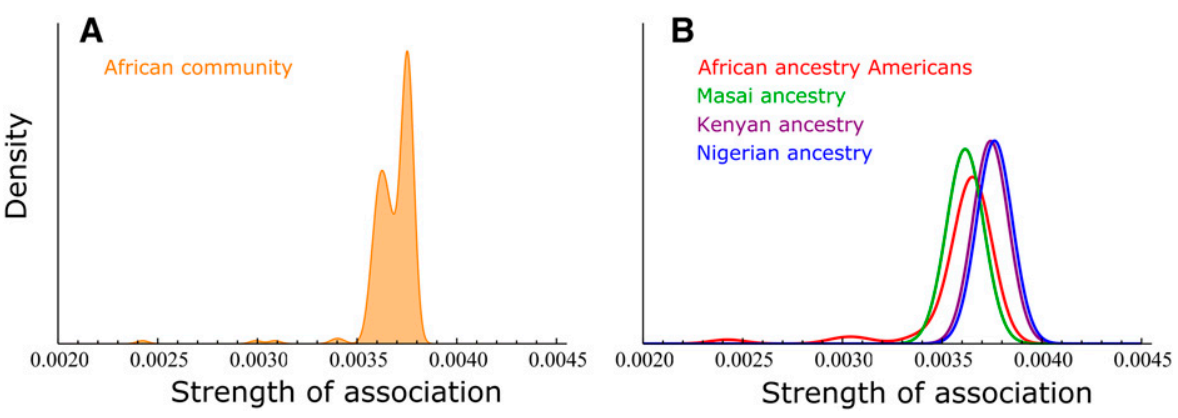

Figure 5 Strength of association distribution (SAD) analysis for the African (orange) community. (A) Distribution of the African community as shown in Figure 2. (B) Distribution of the individuals in the community for different sampled populations. The left mode in $A$ is due to Masai individuals, who were detected as a distinct population by some algorithms (File S1). The African-ancestry American individuals have a slightly lower association with the community than individuals from Nigeria and Kenya, as well as a distinct left tail, perhaps due to recent admixture with people of European or Native American origin. were detected, as for low thresholds in $S_{3}$. The results are summarized in Table 1, with further details given in File S3.

When gene flow was at most medium, the three simulated subpopulations were recovered by community detection, while for high gene flow the procedure was unable to distinguish between $\mathrm{P}_{1}$ and $\mathrm{P}_{2}$. In addition to the detection of subpopulations as the units of structure, the procedure also provided information on hierarchical population structure. For medium gene flow, the low edge-removal thresholds $\mathrm{P}_{1}$ and $\mathrm{P}_{2}$ were detected as one community, $\mathrm{P}_{1} \mathrm{P}_{2}$, and two separate communities for high edge-removal thresholds. The structure at the two hierarchical levels was detected only at intermediate gene flow levels - with low or high gene flow, the same community structure was detected at all thresholds because structure at one hierarchical level was much stronger and masked the structure at the other level.

The $S A$ for all individuals was calculated, and the SAD of the communities was characterized by the mean and coefficient of variation (CV) as a measure of dispersion. The analysis was done for networks with high edge-removal thresholds (before network decomposition), but no significant differences were observed for lower thresholds. While for $S_{1}$ there was no difference in SAD mean of the three detected communities, for the three communities detected in $\mathrm{S}_{2}$ and $S_{3}$ the mean was significantly higher for $\mathrm{P}_{3}$ (File $\mathrm{S} 2$, Figure A). The lower association of individuals from $P_{1}$ and $\mathrm{P}_{2}$ to their assigned communities relative to $\mathrm{P}_{3}$ can be attributed to gene flow between these subpopulations. When two communities were detected, the mean for $\mathrm{P}_{3}$ also was higher than the mean for $\mathrm{P}_{1} \mathrm{P}_{2}$ (File S3, Figure A); however, in this case, it is not possible to tell whether this is a result of gene flow between $\mathrm{P}_{1}$ and $\mathrm{P}_{2}$ or a result of the different number of individuals in $\mathrm{P}_{1} \mathrm{P}_{2}$ and $\mathrm{P}_{3}$. Subpopulations represented by a smaller sample size may show higher $S A$ values due to the fact that common alleles in these subpopulations may be rare in the entire sample (rare alleles in Equation 3 result in edges with higher weights), whereas alleles common in subpopulations with large sample sizes should be relatively common in the entire sample, even if they are rare in other subpopulations.

SAD dispersion also may be an indicator of gene flow because dispersed $S A$ distributions should occur when lineages of many individuals are shared with other subpopulations. While for $S_{2}$ there was no noticeable difference in dispersion between the communities, for $\mathrm{S}_{3}$ (high threshold), the $\mathrm{CV}$ of $\mathrm{P}_{3}$ was significantly smaller than for $\mathrm{P}_{1}$ and $\mathrm{P}_{2}$ (File S3, Table A). For the networks where $\mathrm{P}_{1}$ and $\mathrm{P}_{2}$ were not distinguished $\left(\mathrm{S}_{4}\right.$ and low thresholds in $\left.\mathrm{S}_{3}\right)$, there was no significant difference in dispersion between the communities. Generally, the CV was very low, except for subpopulations experiencing medium gene flow levels, in $\mathrm{S}_{2}$ (File S3, Table A). These results indicate that the high levels of SAD dispersion can be used as an indicator for intermediate levels of gene flow. Overall, the characteristics of the distribution of individual's $S A$, specifically the mean and $\mathrm{CV}$, are correlated with gene flow levels and allow for the distinction among the four simulated scenarios.

\section{Identifying hybrids and recently admixed individuals}

Genetically identifying hybrids or recently admixed individuals of two or more distinct subpopulations is the focus of many studies in ecology and conservation biology (Beaumont et al. 2001; Anderson and Thompson 2002; Allendorf et al. 2012). The simulated scenarios were used to test the ability of SAD analysis to identify hybrids (offspring of individuals from two different subpopulations) and recent descendants of hybrids. In each scenario, a first-generation hybrid, $\mathrm{H}_{1}$, was simulated by randomly selecting one individual from $\mathrm{P}_{1}$ and one individual from $\mathrm{P}_{2}$ and generating a random offspring from the parents' genotypes. Similarly, $\mathrm{H}_{1}$ was backcrossed with a randomly selected individual from $\mathrm{P}_{1}$ to produce a second-generation hybrid, $\mathrm{H}_{2}$. These individuals then were added to the simulated population, and the SAD was analyzed to see whether the hybrid individuals could be identified as outliers (the analysis was done separately for $\mathrm{H}_{1}$ and $\mathrm{H}_{2}$ ). This process was repeated 20 times in all scenarios where the three subpopulations were identified $\left(S_{1}, S_{2}\right.$, and high threshold of $S_{3}$ ).

Because all repeated simulations showed similar results qualitatively, we show only one example of the outlier identification process (Figure 6). For $\mathrm{S}_{1}$ and $\mathrm{S}_{2}$, both $\mathrm{H}_{1}$ and $\mathrm{H}_{2}$ were easily identified as outliers with noticeably lower $S A$ values than the rest of the individuals in their assigned community. For $\mathrm{S}_{3}, \mathrm{H}_{1}$ was revealed as a hybrid with lower $S A$ values, but $\mathrm{H}_{2}$ was not. 
Table 1 Strength of association distribution (SAD) analysis for simulated scenarios

\begin{tabular}{|c|c|c|c|c|c|}
\hline \multicolumn{3}{|c|}{ Scenario parameters } & \multirow[b]{2}{*}{ Detected communities } & \multicolumn{2}{|c|}{ SAD characteristics } \\
\hline Scenario & Gene flow & $m$ & & SAD mean & SAD dispersion \\
\hline$S_{1}$ & None & 0 & $P_{1}, P_{2}, P_{3}$ & Same for all three subpopulations & Same for all three subpopulations \\
\hline $\mathrm{S}_{2}$ & Low & 0.01 & $P_{1}, P_{2}, P_{3}$ & $P_{3}$ has higher mean & Same for all three subpopulations \\
\hline $\mathrm{S}_{3}$ & Medium & 0.05 & $P_{1}, P_{2}, P_{3} a$ & $P_{3}$ has higher mean ${ }^{a}$ & $\mathrm{P}_{3}$ has lower dispersion ${ }^{a}$ \\
\hline & & & $P_{1} P_{2}, P_{3} b$ & $\mathrm{P}_{3}$ has slightly higher mean ${ }^{b}$ & Same for all three subpopulations ${ }^{b}$ \\
\hline $\mathrm{S}_{4}$ & High & 0.1 & $P_{1} P_{2}, P_{3}$ & $P_{3}$ has higher mean & Same for all three subpopulations \\
\hline
\end{tabular}

After the split to three subpopulations, gene flow occurs between $P_{1}$ and $P_{2}$ at rate $m$, and $P_{3}$ remains isolated. Further details are presented in File $S 3$.

${ }^{a}$ High edge-removal thresholds.

${ }^{b}$ Low edge-removal thresholds.

In all cases where $\mathrm{H}_{1}$ or $\mathrm{H}_{2}$ were identified, Equation 2 was examined to determine which $k$ minimized the equation. This was done to identify the second subpopulation with which the hybrid individual was associated. The second population of origin was identified for $\mathrm{H}_{1}$ in all scenarios, while for $\mathrm{H}_{2}$ it was recovered only for low levels of gene flow. Further details regarding identification of hybrid individuals and more detailed results appear in File S3. The results of these simulations show that outliers of $S A$ distributions may be considered to be potential hybrids, but the ability of the method to identify such individuals decreases with decreasing differentiation between the populations in question, as well as with the number of generations after the hybridization event.

\section{Data availability}

File S1 contains details on community detection algorithms and analysis of the human genetic data using various algorithms. File S2 contains the detailed STRUCTURE analysis results on the human genetic data. File S3 contains additional details of the analysis of the simulated data. The Mathematica code for running NetStruct can be found at giligreenbaum. wordpress.com/software and github.com/GiliG/NetStruct.

\section{Discussion}

We present a model-free distance-based approach for analysis of population structure at the individual-subpopulation level that does not entail the assumption of an underlying model or any prior conditions. The approach is set in a network theory framework and uses the concepts of community and modularity, which allow for computationally efficient assignment of individuals to subpopulations. The computational efficiency also makes this approach applicable in cases where many loci are studied. An additional exploratory analysis of the $S A$ distributions of the communities can be used to study population structure beyond assignment of individuals to populations by evaluating the strength with which individuals are associated with their assigned subpopulations. This may be useful to detect hybrid and recently admixed individuals, as well as to explore finer details of the population structure, as demonstrated by analysis of simulated and real data sets.
Clustering individuals based on genetic composition has become important for many studies in various fields, such as ecology, conservation, medicine, and anthropology, and there are now several methods and programs addressing this task (Waples and Gaggiotti 2006; Wollstein and Lao 2015). The more developed approach is the model-based approach, which uses Bayesian or maximum-likelihood methods to estimate allele frequencies of $K$ hypothetical populations, under model assumptions such as HWE, and assigns probabilities of assignment for individuals to these populations. The alternative, model-free, approach is based on analysis of distance (dissimilarity) or similarity matrices, which summarize some measure of distance or similarity between all pairs of individuals. These matrices are usually analyzed using MDS such as PCA and projected to two or three coordinates for visualization [e.g., PLINK (Purcell et al. 2007), EIGENSOFT (Price et al. 2006), and GENEALEX (Peakall and Smouse 2006, 2012)]. Clusters are determined either visually or using other clustering methods, such as $k$-means (MacQueen 1967), often requiring a priori definition of the number of clusters to be found, $K$. Similarly, other distance-based methods apply spectral clustering techniques and analyze the eigenvalue spectra of similarity matrices (Lee et al. 2010). Because model- and distance-based approaches differ significantly in methodology and prior assumptions, researchers often apply both approaches to the same data set to ensure that interpretations are not biased by methodology.

The network approach we present here falls under the distance-based clustering category, and no underlying model is assumed. Clustering is done by locating groups of nodes that are strongly connected within the group but weakly connected to nodes outside the group. The strength of the intracommunity connections is not defined by a fixed value but rather is determined relative to the network structure. For example, spectral community-detection methods (Newman 2006, 2013) do not analyze the spectra of the similarity matrix itself but rather address the modularity matrix, which is constructed by comparing the strength of connection between nodes with what would be expected in a random network with similar structural properties (i.e., same node degrees). The community-detection methods used in our approach therefore differ from other distance-based clustering methods in what is considered a "good cluster" and have been 
H1
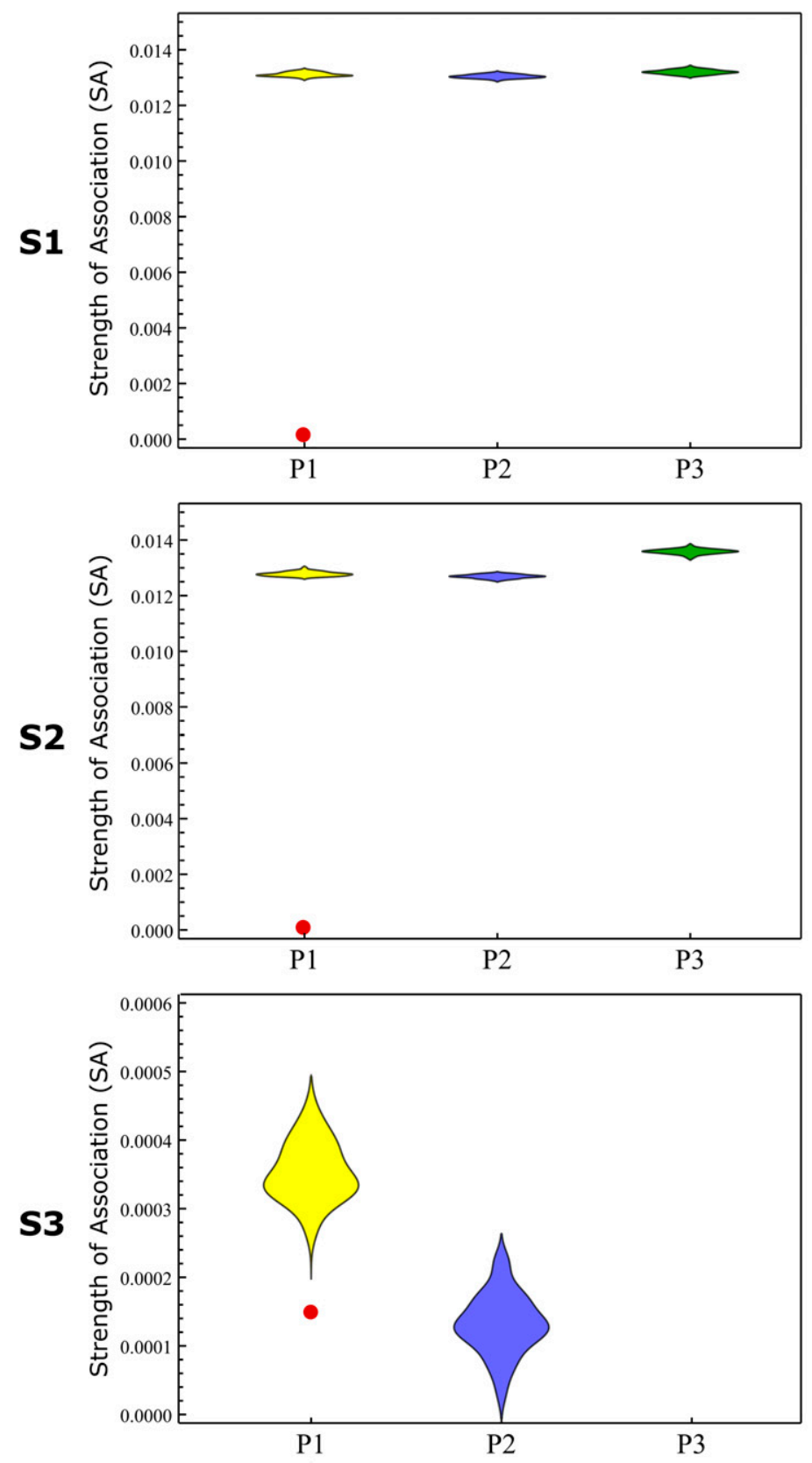

H2
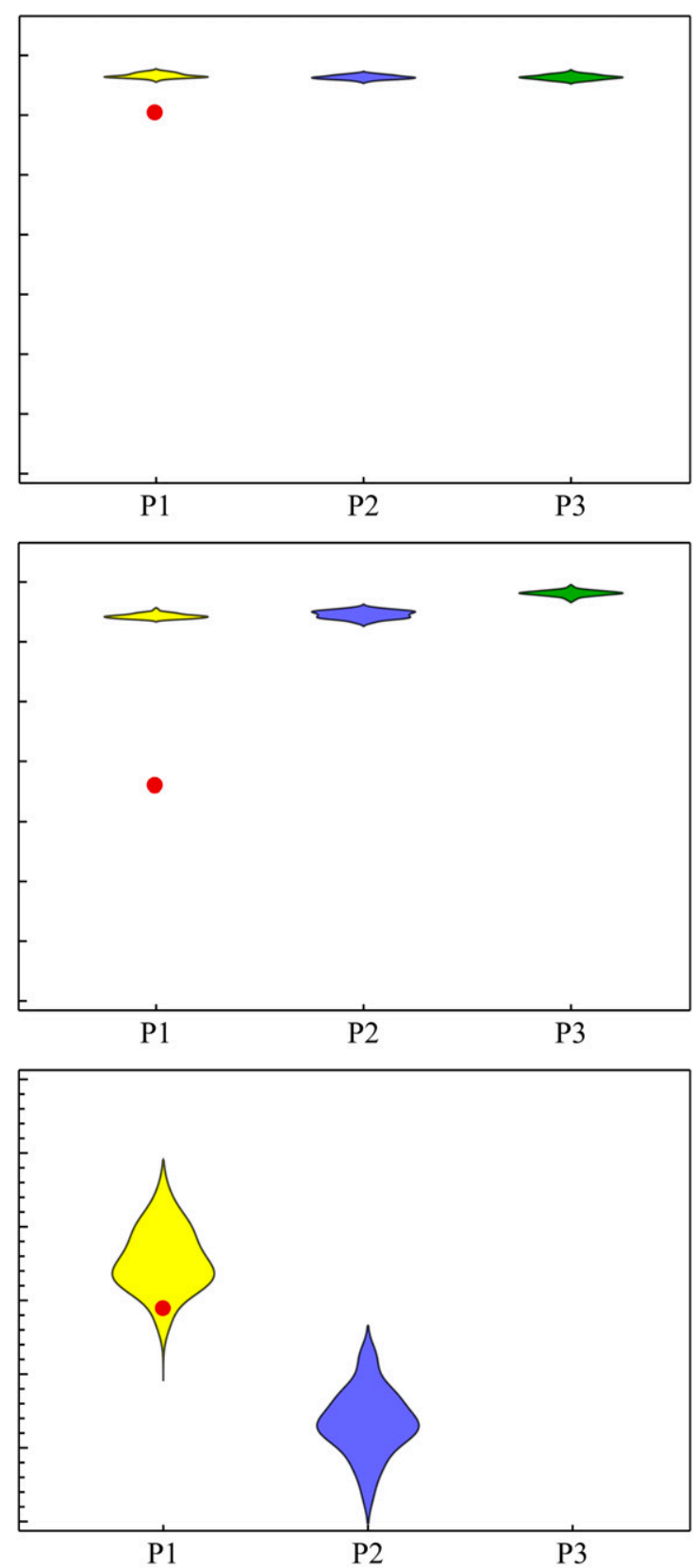

Figure 6 An example of hybrid identification using strength of association distribution (SAD) analysis. Distribution charts show the SAD for the three detected communities, with the $S A$ of the hybrid shown as a red dot (distribution of $P_{3}$ in $S_{3}$ is out of scale). The analysis was performed on simulated scenarios $S_{1}, S_{2}$, and $S_{3}$, where $H_{1}$ is a hybrid between populations $P_{1}$ and $P_{2}$, and $H_{2}$ is a second-generation hybrid between $H_{1}$ and $P_{1}$. Hybrids are identified as low SAD outliers in all cases except for $\mathrm{H}_{2}$ in $\mathrm{S}_{3}$.

shown to detect meaningful clusters in many systems where clusters are better defined by relative relations between elements than by other distance-based definitions (Girvan and Newman 2002; Fortunato 2010). In this sense, community detection is more similar to model-based approaches, but here no prior assumptions are required. This also makes it more natural to weigh pairwise genetic similarity by allele frequencies to strengthen similarity between individuals sharing rare alleles relative to those sharing common alleles
(Equation 3), whereas other distance-based methods more commonly employ only identity-by-state (IBS) measures to define similarity or distance between individuals.

Ideally, population-level studies would benefit from exploring structure using our network approach in combination with model-based clustering methods and MDS visualization since these complement each other and may give a more robust and detailed picture. In the example analyzed in this paper using human SNP data, the model-based analysis and 
the network analysis were relatively in agreement regarding assignment of individuals to subpopulations. However, the network analysis did detect the difference between the Chinese and Japanese groups, which was not detected by the software STRUCTURE. Additionally, the SAD analysis revealed differences in gene flow experienced by the Masai and African-ancestry American groups, but these groups appear very similar in the STRUCTURE analysis (Figure 3 ).

The STRUCTURE analysis did indicated biologically meaningful structure at different hierarchical levels, but this was done using a heuristic, and the hypothesis of population structure at any hierarchical level is not statistically testable. Statistically testable hierarchical partitioning of population structure is, of course, feasible and common in subpopulation/ population-level methods (e.g., F-statistics and AMOVA) but is not common in individual-subpopulation procedures, where putative subpopulations are not defined a priori. With the approach presented here, a similar hierarchical structure was detected through systematic examination of edgeremoval thresholds, but here each hierarchical level was statistically tested and shown to be significant. Statistically testing hierarchical structure is important because otherwise analyses are forced either to recover only the strongest level, missing biologically relevant information about the system, or to report many hierarchical levels, without a procedure for determining which are meaningful and which are not. With the network method presented here, different significant community structures emerge, producing a semihierarchical structure in the sense that a community partition at a given level does not depend on "higher-level" partitions. True hierarchical community partition procedures (Palla et al. 2005; Guimera et al. 2007) can possibly be useful for hierarchical population structure analysis, but in most of these procedures, each level is constrained by higher levels.

Another related issue that has been a concern in modelbased implementation is the assessment of the number of subpopualtions $K$ (Pritchard et al. 2000; Evanno et al. 2005) because $K$ is usually one of the model parameters. This problem also arises when applying clustering techniques to results of distance-based methods, such as $k$-means on MDS results. By setting $K$, these procedures regard the subpopulations as equivalent, even though this is often not the case. For example, for the network shown in Figure $1 \mathrm{~B}, K=3$, but the three subpopulations show very different distributions of withinpopulation relatedness (Figure 2). In the network-based approach, there is no such issue because most communitydetecting methods do not a priori assume $K$ but rather find the optimal $K$ that maximizes modularity (e.g., Clauset et al. 2004; Newman 2004b) or acquire $K$ as part of the detection process (e.g., Pons and Latapy 2006; Newman 2006; Raghavan et al. 2007), without assuming any equivalence of the communities. This is due to the fact that modularity is a trusted and well-studied measure for the quality of community partitions (Newman 2004b).

With whole-genome sequencing becoming more and more accessible, procedures for population structure analysis also must take into account computational considerations. The procedure presented here is composed of three consecutive steps, with construction of the network taking $O\left(L n^{2}\right)$ time, where $n$ is the number of individuals in the sample, and $L$ is the number of loci involved in the analysis. Computation time for community detection depends on the algorithm used, but fast near-linear algorithms, taking $O(n+m)$ time, where $m$ is the number of edges in the network, and approaching $O(n)$ time for sparse networks (which can be constructed using high enough edge-removal thresholds), are already available (Raghavan et al. 2007). SAD analysis depends on $m$ and on the number of communities detected $c$ and takes $O(\mathrm{cnm})$ time, approaching $O\left(\mathrm{cn}^{2}\right)$ for sparse networks. Only the first step involves $L$; therefore, the computation time of the entire procedure is linear with respect to the number of loci, and there should be no computational limitation for including full-genome sequences in analyses.

Because the genetic-similarity measure, the threshold, and the community-detection algorithm remain, for now, user defined and may result in different population structures, care must be taken when defining these parameters, and preferably several options should be explored. Further studies may provide guidelines for setting these parameters as a function of the particulars of the system under study. Network theory, particularly community detection, is a highly active field of research, but our understanding of the usefulness of particular community-detection procedures to different types of networks is still minimal, and future advancements in network theory may provide clearer guidelines for algorithm and threshold choice. One possible direction for formalizing the systematic edge-removal threshold procedure could be by drawing ideas from persistence homology theory (Edelsbrunner and Harer 2008), except that here we are interested in the persistence of the modular structures of networks over different thresholds rather than the persistence of homology groups of simplicial complexes. As with most other individual-subpopulation level clustering methods, significant linkage disequilibrium (LD) between loci should be avoided, and data should be screened to ensure that loci can be regarded as independent. The extent to which LD will affect both community partition and the significance test presented here also should be explored in the future.

We believe that a network approach may provide an additional complementary viewpoint on population structure analysis, one less hampered by prior assumptions. Moreover, defining problems in population genetics in network terminology is important for establishing the usage of network theory in population genetics. Currently, many tools and methods are developed within network theory to study complex systems, and these methods may become accessible to the field of population genetics once network terminology is appropriately incorporated in population genetics theory and practice.

The method presented here is implemented in the program NetStruct, which uses community-detection algorithms implemented in the software package igraph (Csárdi and 
Nepusz 2006) and is available at https://giligreenbaum. wordpress.com/software/.

\section{Acknowledgments}

The authors thank Yair Zarmi, Sharon Renan, Naama Shahar, Amos Bouskila and Shai Pilosof for insightful comments and discussions. This research was supported by a United StatesIsrael Binational Science Foundation grant (2011384) awarded to S.B., A.R.T., and Amos Bouskila. This is publication 894 of the Mitrani Department of Desert Ecology.

\section{Literature Cited}

Alexander, D. H., J. Novembre, and K. Lange, 2009 Fast modelbased estimation of ancestry in unrelated individuals. Genome Res. 19: 1655-1664.

Allendorf, F. W., G. H. Luikart, and S. N. Aitken, 2012 Conservation and the genetics of populations. Wiley-Blackwell, Hoboken, NJ.

Anderson, E. C., and E. a. Thompson, 2002 A model-based method for identifying species hybrids using multilocus data. Genetics 160: 1217-1229.

Ball, M. C., L. Finnegan, M. Manseau, and P. Wilson, 2010 Integrating multiple analytical approaches to spatially delineate and characterize genetic population structure: an application to boreal caribou (Rangifer tarandus caribou) in central canada. Conserv. Genet. 11: 2131-2143.

Beaumont, M., E. M. Barratt, D. Gottelli, C. Kitchener, M. J. Daniels et al., 2001 Genetic diversity and introgression in the scottish wildcat. Mol. Ecol. 10: 319-336.

Blatt, M., S. Wiseman, and E. Domany, 1996 Superparamagnetic clustering of data. Phys. Rev. Lett. 76: 3251-3254.

Burren, A., H. Signer-Hasler, M. Neuditschko, J. Tetens, J. Kijas et al., 2014 Fine-scale population structure analysis of seven local swiss sheep breeds using genome-wide snp data. Anim. Genet. Resour. 55: 67-76.

Cavalli-Sforza, L. L., and A. W. Edwards, 1967 Phylogenetic analysis. models and estimation procedures. Am. J. Hum. Genet. 19: 233-257.

Clauset, A., M. E. J. Newman, and C. Moore, 2004 Finding community structure in very large networks. Phys. Rev. E Stat. Nonlin. Soft Matter Phys. 70: 1-6.

Cohen, O., A. Barocas, and E. Gefen, 2013 Conflicting management policies for the Arabian wolf Canis lupus arabs in the Negev Desert: is this justified? Oryx 47: 228-236.

Corander, J., P. Waldmann, and M. J. Sillanpää, 2003 Bayesian analysis of genetic differentiation between populations. Genetics 163: 367-374.

Csárdi, G., and T. Nepusz, 2006 The igraph software package for complex network research. InterJournal 2006: 1695.

Dyer, R. J., and J. D. Nason, 2004 Population graphs: the graph theoretic shape of genetic structure. Mol. Ecol. 13: 1713-1727.

Edelsbrunner, H., and J. Harer, 2008 Persistent homology: a survey, pp. 257-281 in Surveys on Discrete and Computational Geometry: Twenty Years Later (Contemporary Mathematics Series, Vol. 453), edited by J. E. Goodman, J. Pach, and R. Pollack. American Mathematical Society, Providence, RI.

Evanno, G., S. Regnaut, and J. Goudet, 2005 Detecting the number of clusters of individuals using the software structure: A simulation study. Mol. Ecol. 14: 2611-2620.

Excoffier, L., P. E. Smouse, and J. M. Quattro, 1992 Analysis of molecular variance inferred from metric distances among DNA haplotypes: application to human mitochondrial DNA restriction data. Genetics 131: 479-491.

Forst, C. V., 2002 Network genomics: a novel approach for the analysis of biological systems in the post-genomic era. Mol. Biol. Rep. 29: 265-280.

Fortunato, S., 2010 Community detection in graphs. Phys. Rep. 486: 75-174.

Garroway, C. J., J. Bowman, D. Carr, and P. J. Wilson, 2008 Applications of graph theory to landscape genetics. Evol. Appl. 1: 620-630.

Girvan, M., and M. E. J. Newman, 2002 Community structure in social and biological networks. Proc. Natl. Acad. Sci. USA 99: 7821-7826.

Guimera, R., A. Moreira, D. Rodrigues, E. Mar, R. Bateman et al., 2007 Extracting the hierarchical organization. Proc. Natl. Acad. Sci. USA 104: 15224-15229.

International HapMap Consortium, 2003 The International HapMap Project. Nature 426: 789-796.

Jombart, T., S. Devillard, A.-B. Dufour, and D. Pontier, 2008 Revealing cryptic spatial patterns in genetic variability by a new multivariate method. Heredity 101: 92-103.

Lancichinetti, A., and S. Fortunato, 2009 Community detection algorithms: A comparative analysis. Phys. Rev. E 80: 056117.

Laval, G., and L. Excoffier, 2004 SIMCOAL 2.0: a program to simulate genomic diversity over large recombining regions in a subdivided population with a complex history. Bioinformatics 20: 2485-2487.

Lee, A. B., D. Luca, L. Klei, B. Devlin, and K. Roeder, 2010 Discovering genetic ancestry using spectral graph theory. Genet. Epidemiol. 34: 51-59.

Li, C. C., and D. G. Horvitz, 1953 Some methods of estimating the inbreeding coefficient. Am. J. Hum. Genet. 5: 107-117.

MacQueen, J. B., 1967 Some methods for classification and analysis of multivariate observations, pp. 281-297 in Proceedings of the 5th Berkeley Symposium on Mathematical Statistics and Probability, Vol. 1. University of California Press, Berkeley, CA.

Munwes, I., E. Geffen, U. Roll, A. Friedmann, A. Daya et al., 2010 The change in genetic diversity down the core-edge gradient in the eastern spadefoot toad (pelobates syriacus). Mol. Ecol. 19: 2675-2689.

Neuditschko, M., M. S. Khatkar, and H. W. Raadsma, 2012 NetView: a high-definition network-visualization approach to detect fine-scale population structures from genomewide patterns of variation. PLoS One 7: e48375.

Neuditschko, M., M. S. Khatkarm, and H. W. Raadsma, 2014 Fine scale population structure of global cattle breeds using dense haplotype data. In Proceedings of the 10th World Congress of Genetics Applied to Livestock Production. American Society of Animal Science, Champaign, IL. (Abstract) 166: 1-3.

Newman, M., 2006 Modularity and community structure in networks. Proc. Natl. Acad. Sci. USA 103: 8577-8582.

Newman, M. E. J., 2004a Analysis of weighted networks. Phys. Rev. E Stat. Nonlin. Soft Matter Phys. 70: 1-9.

Newman, M. E. J., 2004b Detecting community structure in networks. Eur. Phys. J. B 38: 321-330.

Newman, M. E., 2013 Spectral methods for community detection and graph partitioning. Phys. Rev. E Stat. Nonlin. Soft Matter Phys. 88: 42822.

Newman, M. E. J., and M. Girvan, 2004 Finding and evaluating community structure in networks. Phys. Rev. E Stat. Nonlin. Soft Matter Phys. 69: 026113.

Oliehoek, P. A., J. J. Windig, J. A. M. Van Arendonk, and P. Bijma, 2006 Estimating relatedness between individuals in general populations with a focus on their use in conservation programs. Genetics 173: 483-496. 
Opsahl, T., and P. Panzarasa, 2009 Clustering in weighted networks. Soc. Networks 31: 155-163.

Palla, G., I. Derényi, I. Farkas, and T. Vicsek, 2005 Uncovering the overlapping community structure of complex networks in nature and society. Nature 435: 814-818.

Peakall, R., and P. E. Smouse, 2006 GENALEX 6: genetic analysis in excel. Population genetic software for teaching and research. Mol. Ecol. Notes 6: 288-295.

Peakall, R., and P. E. Smouse, 2012 GENALEX 6.5: genetic analysis in Excel. Population genetic software for teaching and research-an update. Bioinformatics 28: 2537-2539.

Pickrell, J. K., and J. K. Pritchard, 2012 Inference of population splits and mixtures from genome-wide allele frequency data. PLoS Genet. 8: e1002967.

Pons, P., and M. Latapy, 2006 Computing communities in large networks using random walks. J. Graph Algorithms Appl. 10: 191-218.

Price, A. L., N. J. Patterson, R. M. Plenge, M. E. Weinblatt, N. A. Shadick et al., 2006 Principal components analysis corrects for stratification in genome-wide association studies. Nat. Genet. 38: 904-909.

Pritchard, J. K., M. Stephens, and P. Donnelly, 2000 Inference of population structure using multilocus genotype data. Genetics 155: 945-959.

Purcell, S., B. Neale, K. Todd-Brown, L. Thomas, M. A. R. Ferreira et al., 2007 PLINK: a tool set for whole-genome association and population-based linkage analyses. Am. J. Hum. Genet. 81: 559-575.

Raghavan, U. N., R. Albert, and S. Kumara, 2007 Near linear time algorithm to detect community structures in large-scale networks. Phys. Rev. E 76: 1-11.

Rozenfeld, A. F., S. Arnaud-Haond, E. Hernández-García, V. M. Eguíluz, E. A. Serrão et al., 2008 Network analysis identifies weak and strong links in a metapopulation system. Proc. Natl. Acad. Sci. USA 105: 18824-18829.

Saitou, N., and M. Nei, 1987 The neighbor-joining method: a new method for reconstructing phylogenetic trees. Mol. Biol. Evol. 4: 406-425.

Templeton, A., 2006 Population Genetics and Microevolutionary Theory. Wiley, Hoboken, NJ.

Waples, R. S., and O. Gaggiotti, 2006 What is a population? An empirical evaluation of some genetic methods for identifying the number of gene pools and their degree of connectivity. Mol. Ecol. 15: 1419-1439.

Wollstein, A., and O. Lao, 2015 Detecting individual ancestry in the human genome. Investig. Genet. 6: 1-12.

Yang, W.-Y., J. Novembre, E. Eskin, and E. Halperin, 2012 A model-based approach for analysis of spatial structure in genetic data. Nat. Genet. 44: 725-731.

Communicating editor: $N$. H. Barton 


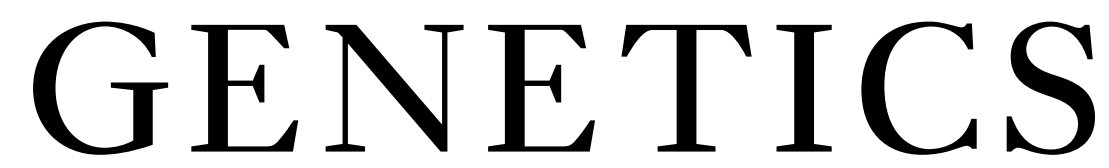

Supporting Information www.genetics.org/lookup/suppl/doi:10.1534/genetics.115.182626/-/DC1

\section{Inference and Analysis of Population Structure Using Genetic Data and Network Theory}

Gili Greenbaum, Alan R. Templeton, and Shirli Bar-David 


\section{File S1}

\section{Using different community detection algorithms for analysis of human genetic data}

A community in a network is a dense subgraph within the network. However, there are many different ways to partition a network into subgraphs, and there is no clear way to determine which partition is a better description of a division of the network to dense subgraphs, i.e. communities. The concept of modularity has been shown to correlate well with sensible partitions of well studied networks, and is thus generally regarded as a good measure for the quality of a given community partition[1]. However, finding a community partition that maximizes the modularity is a very complicated computational task, and many algorithms have been proposed in order to efficiently approximate such a partition. Moreover, other non-modularity based approaches have been proposed for detecting community structure in networks.

In this appendix we use five commonly used community detection algorithms, implemented in the software package igraph[2] as an alternative to the Girvan-Newman community detection algorithm presented in the main text. We produce figures analogous to Figure 1 in the main text (Figures A-E) using the following algorithms: 1) Label Propagation[3] - a stochastic algorithm where labels attached to nodes are iterated until a consensus is reached. The groups with the same label at consensus are the detected communities 2) Walktrap [4] - an algorithm based on random walks on graphs. Random walks should tend to get "trapped" more often within communities, and this is used to identify community structure 3)FastGreedy[5] - A greedy algorithm version of the GirvanNewman algorithm 4)Leading Eigenvector[6] - an algorithm assigning individuals to communities based on the properties of the leading eigenvector of the modularity matrix 5)Spinglass[7] - an algorithm derived from statistical mechanics. The thresholds were determined by systematically exploring the parameter space in a similar manner as was done for the Girvan-Newman algorithm, detailed in the main text (Methods and Analysis of human SNP data sections). The specific values may differ for different algorithms as the threshold ranges with different partitions depend on the specific algorithm used.

The community structure pattern revealed using different algorithms is similar to that when using the Girvan-Newman algorithm (all community structures where significant at $p \leq 0.001$ ). The only clear difference is that some algorithms (Girvan-Newman, Label Propagation; Fig 1 in the main text, Fig. A) detect 6 subpopulations when using the high thresholds, while other algorithms (Walktrap, FastGreedy, Spinglass, Leading Eigenvector; Fig B-E) detect 7 communities at those thresholds. The additional community (green nodes) corresponds to the Masai population. 


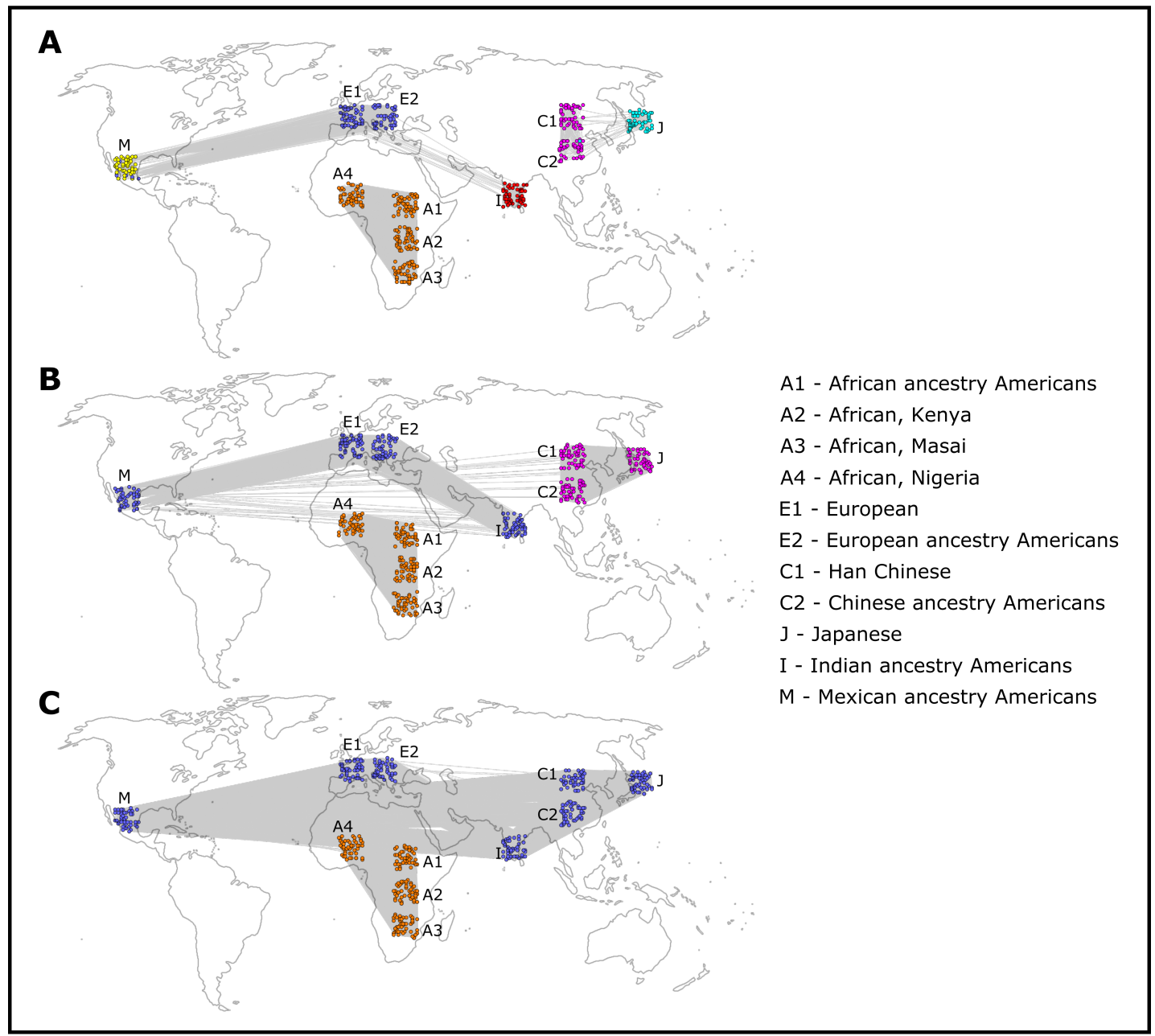

Figure A: Community detection using Label propagation algorithm on three networks with different thresholds. Each node represents an individual, with colors representing the community assigned by the algorithm. For visualization purpose, individuals are placed on the world map roughly corresponding to their ancestry.A) Threshold 0.198, 0.208 for East Asian component B)Threshold 0.195 C) Threshold 0.187. 


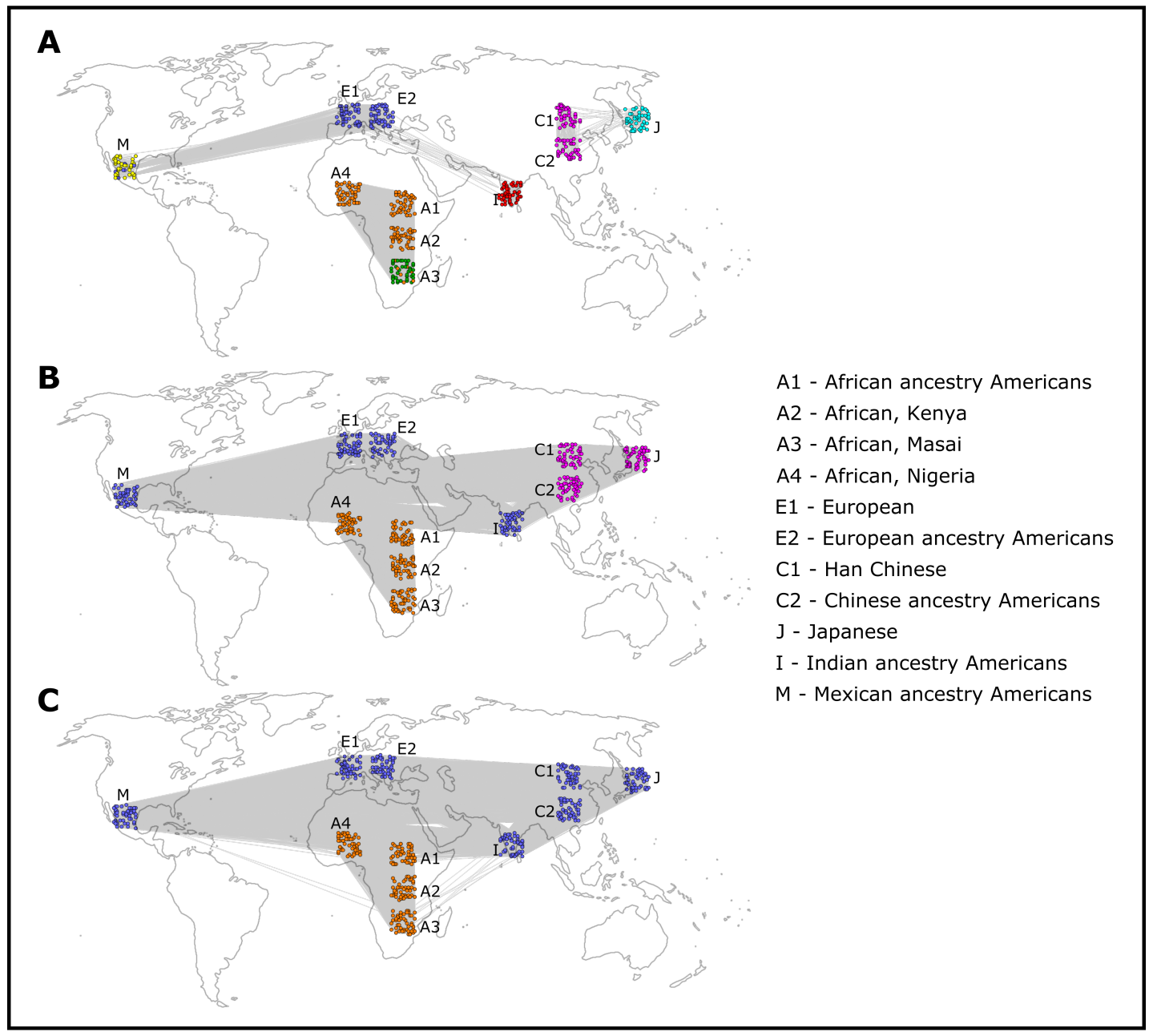

Figure B: Community detection using Walktrap algorithm on three networks with different thresholds. Each node represents an individual, with colors representing the community assigned by the algorithm. For visualization purpose, individuals are placed on the world map roughly corresponding to their ancestry.A) Threshold 0.198, 0.208 for East Asian component B)Threshold 0.197 C) Threshold 0.189. 


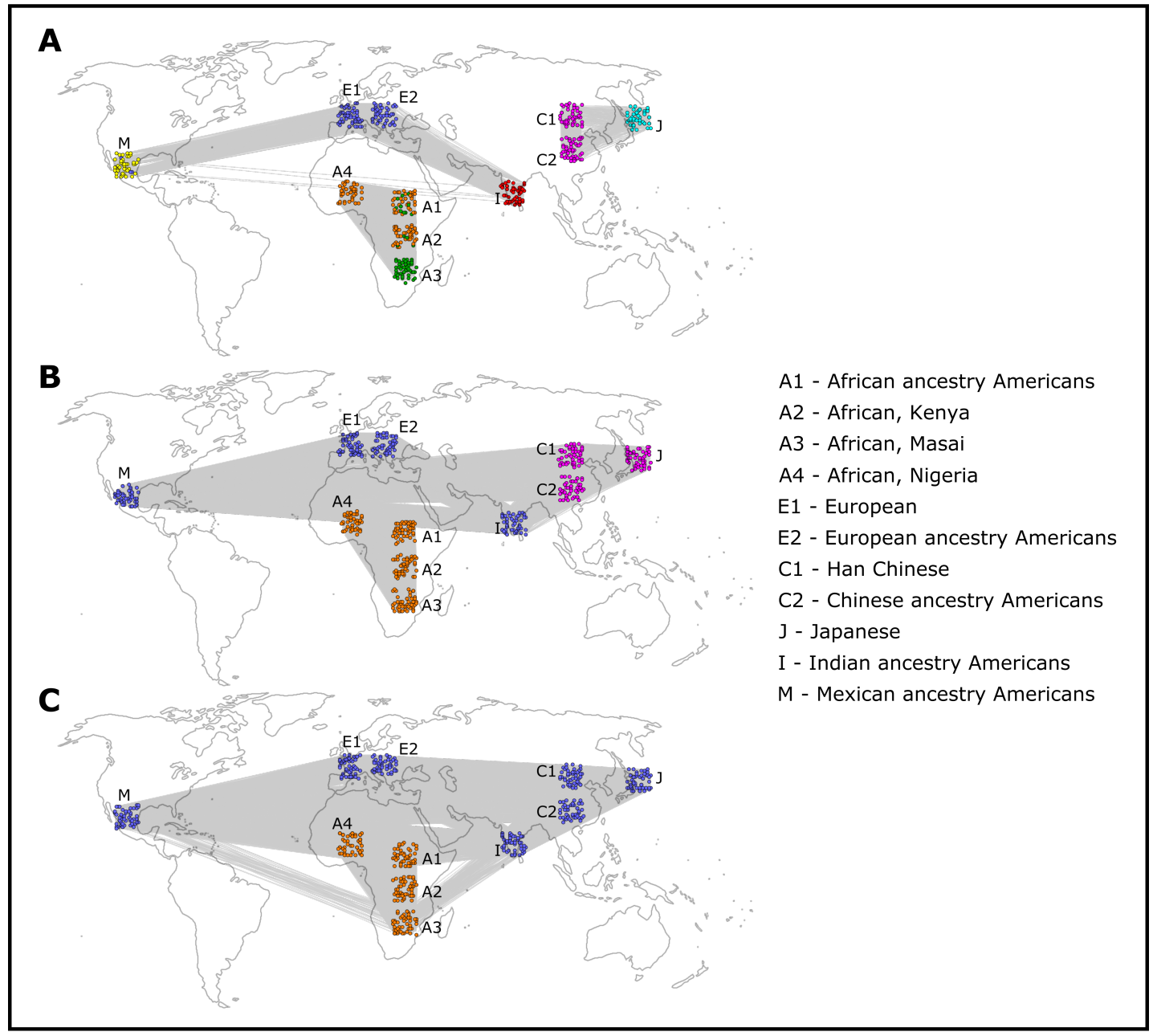

Figure C: Community detection using FastGreedy algorithm on three networks with different thresholds. Each node represents an individual, with colors representing the community assigned by the algorithm. For visualization purpose, individuals are placed on the world map roughly corresponding to their ancestry.A) Threshold 0.196, 0.208 for East Asian component B)Threshold 0.193 C) Threshold 0.189. 


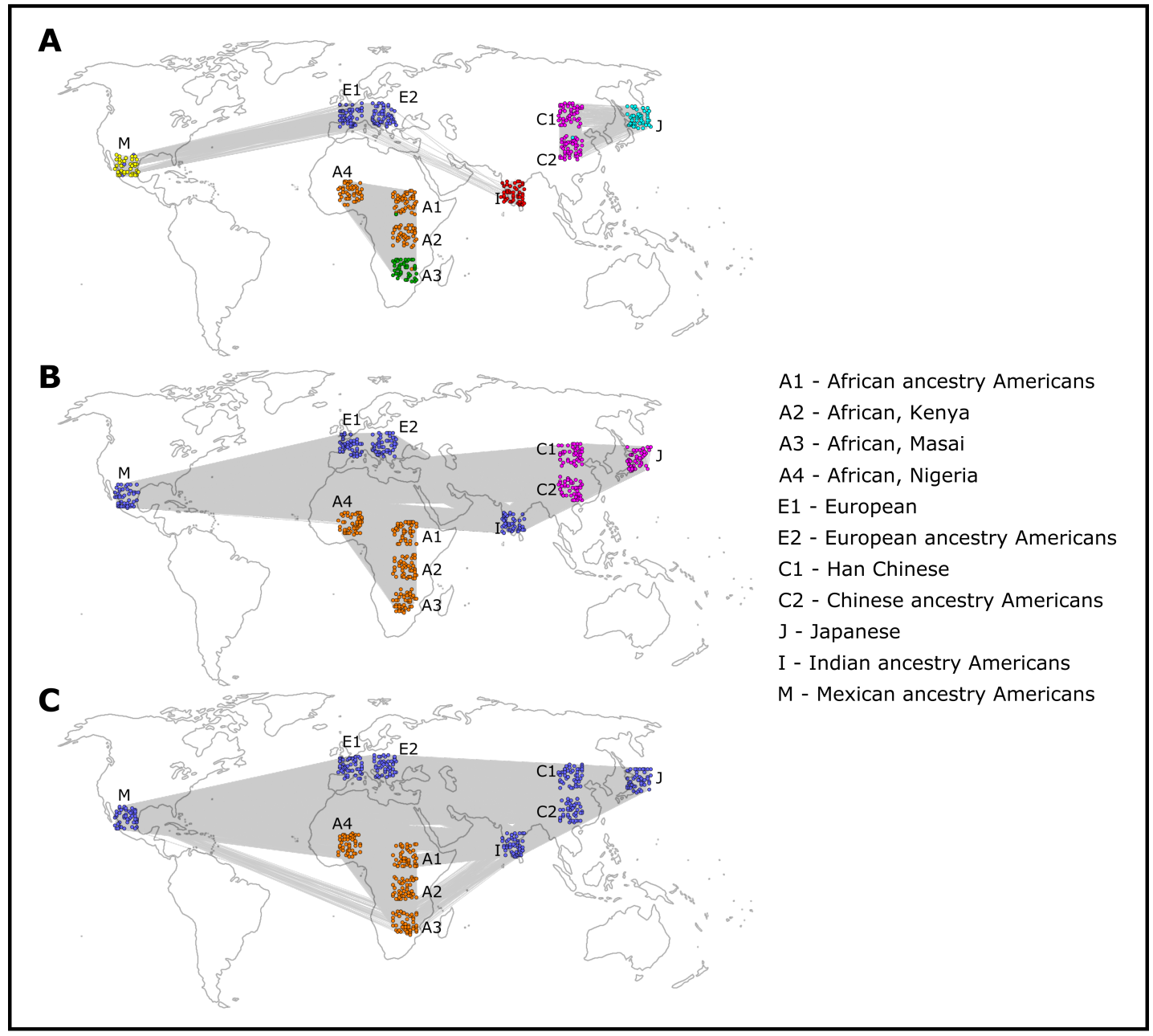

Figure D: Community detection using Leading Eigenvector on three networks with different thresholds. Each node represents an individual, with colors representing the community assigned by the algorithm. For visualization purpose, individuals are placed on the world map roughly corresponding to their ancestry.A) Threshold 0.198, 0.207 for East Asian component B)Threshold 0.188 C) Threshold 0.184. 


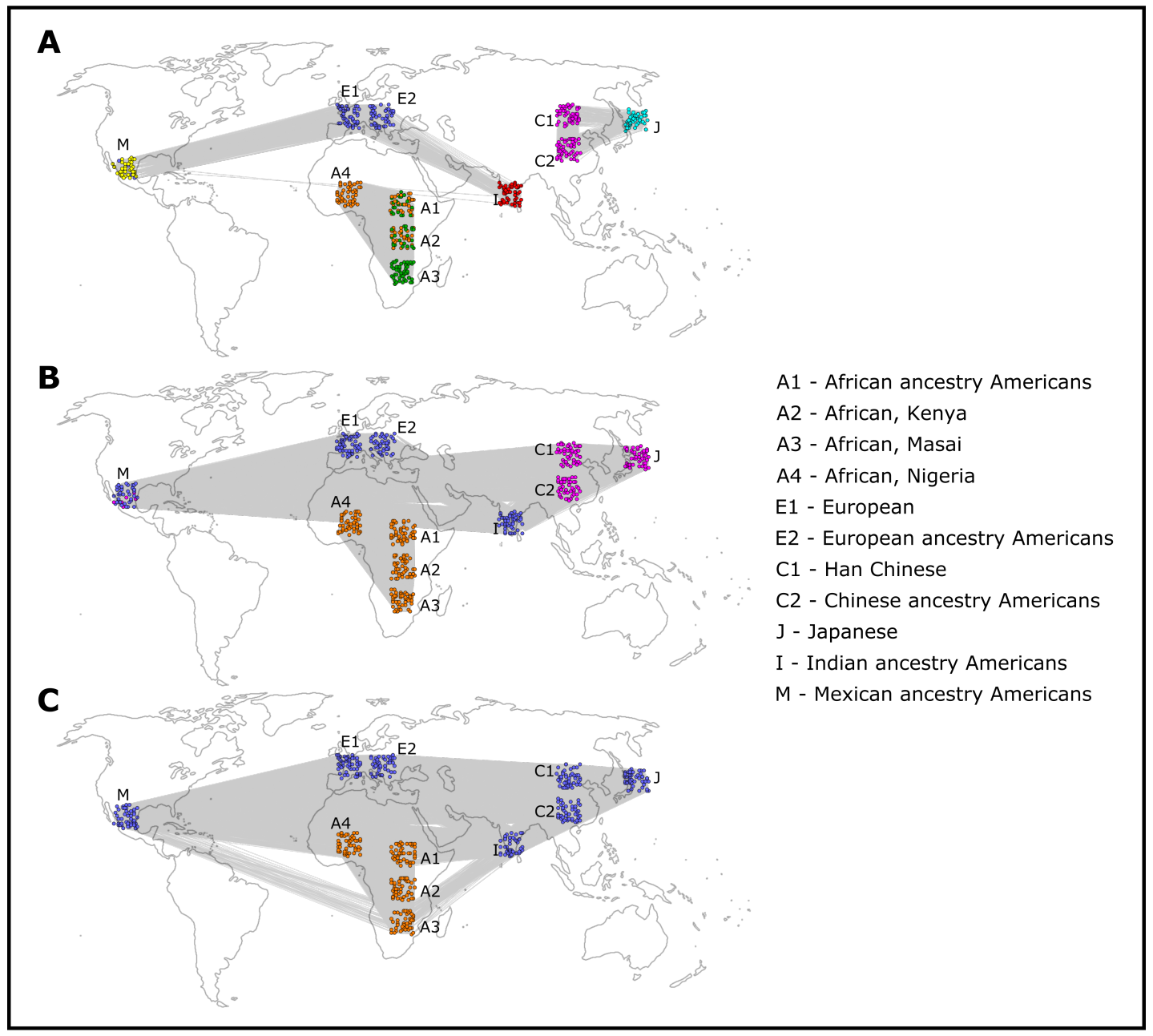

Figure E: Community detection using Spinglass algorithm on three networks with different thresholds. Each node represents an individual, with colors representing the community assigned by the algorithm. For visualization purpose, individuals are placed on the world map roughly corresponding to their ancestry.A) Threshold 0.197, 0.207 for East Asian component B)Threshold 0.189 C) Threshold 0.184. 


\section{References}

[1] M. E. J. Newman. "Detecting community structure in networks". In: The European Physical Journal B 38.2 (2004), pp. 321-330.

[2] G Csárdi and T Nepusz. "The igraph software package for complex network research". In: InterJournal Complex Systems 1695 (2006), p. 1695.

[3] U. N. Raghavan, R. Albert, and S. Kumara. "Near linear time algorithm to detect community structures in large-scale networks". In: Physical Review E - Statistical, Nonlinear, and Soft Matter Physics 76.3 (2007), pp. 1-11.

[4] P. Pons and M. Latapy. "Computing Communities in Large Networks Using Random Walks". In: Computer and Information Sciences - ISCIS 2005. Berlin, Germany: Springer, 2005, pp. 284293.

[5] A. Clauset, M. E. J. Newman, and C. Moore. "Finding community structure in very large networks". In: Physical Review E - Statistical, Nonlinear, and Soft Matter Physics 70.066111 (2004), pp. 1-6.

[6] M. Newman. "Modularity and community structure in networks". In: Proceedings of the National Academy of Science 103 (2006), pp. 8577-8582.

[7] J. Reichardt and S. Bornholdt. "Statistical mechanics of community detection". In: Physical Review E - Statistical, Nonlinear, and Soft Matter Physics 74.1 (2006), pp. 1-14. 


\section{File S2}

\section{Model-based analysis of human genetic data using STRUCTURE}

The human genetic data were analyzed using the software STRUCTURE[1]. For this the Admixture model was used without the LocPrior option, with 10,000 burnin period and 10,000 iterations. First, from computation time considerations, 1000 SNPs were randomly subsampled, and 10 repeats for each $K$ value from 1 to 11 were performed. These data were analyzed with the software STRUCTURE HARVESTER[2] to generate the DeltaK values according to the heuristic method introduced by Evanno[3], shown in Figure A. The most likely $K$ according to this analysis is $K=2$, but the relatively high DeltaK values for $K=3$ and $K=6$ indicates that these are also likely options. This process was repeated 5 times with different 1000 SNP subsamples, with similar results. Following this, the STRUCTURE analysis was performed again, with the same parameters but without repeats for different $K$ values, using all 22,000 SNPs for $K=2,3$ and 6 . The results are shown in Figure B.

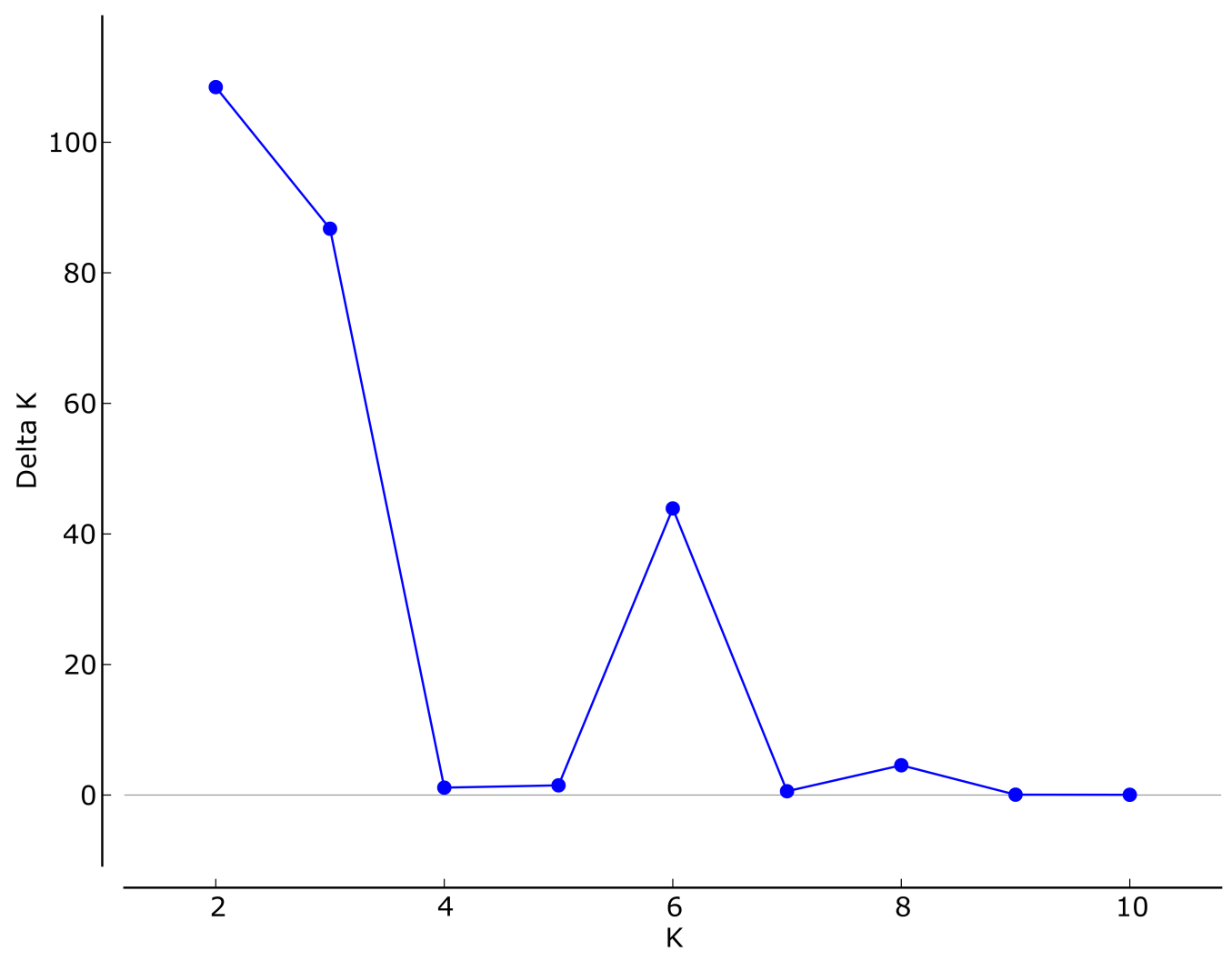

Figure A: DeltaK plot following the Evanno method. 

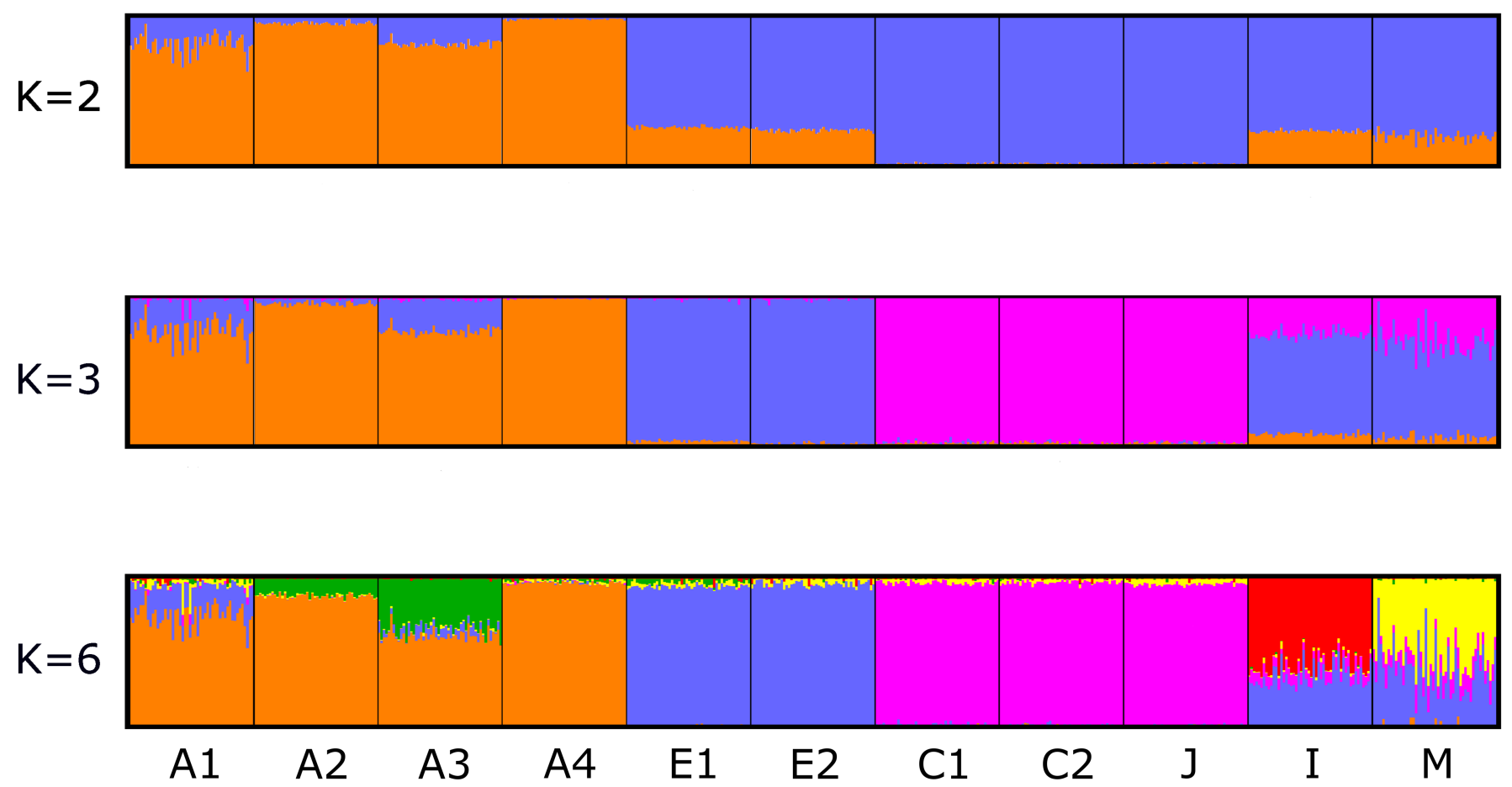

Figure B: Model-based analysis of human SNP data using the program STRUCTURE. Results assuming $K=2, K=3$ and $K=6$. Sampled populations: A1 - African ancestry Americans; A2 African (west Kenya); A3 - African (Masai); A4 - African (Nigeria); E1 - Europeans; E2 - European ancestry Americans; C1 - Han Chinese; C2 - Chinese ancestry Americans; J - Japanese; I - Indian ancestry Americans; M - Mexican ancestry Americans. 


\section{References}

[1] J. K. Pritchard, M Stephens, and P Donnelly. "Inference of population structure using multilocus genotype data." In: Genetics 155.2 (2000), pp. 945-959.

[2] D. a. Earl and B. M. VonHoldt. "STRUCTURE HARVESTER: A website and program for visualizing STRUCTURE output and implementing the Evanno method". In: Conservation Genetics Resources 4.2 (2012), pp. 359-361.

[3] G. Evanno, S. Regnaut, and J. Goudet. "Detecting the number of clusters of individuals using the software STRUCTURE: A simulation study". In: Molecular Ecology 14.8 (2005), pp. 26112620 . 


\section{File S3 - Analyses of simulated scenarios}

\section{Strength of Association Distribution (SAD) analysis}

SAD analysis was performed on the four scenarios detailed in the main text. Community detection, using the FastGreedy algorithm, was applied for edge-removal thresholds starting from 0 in steps of 0.01 until the network was decomposed to many disconnected components. Decomposition of the network occurred at threshold 0.07 for $\mathrm{S} 1$ and 0.06 for the other scenarios. The significance of the partitions was tested using the permutation test described in the main test with 1000 permutations, and all community structures were found to be significant $(p \leq 0.001)$. Table A details the Coefficient of Variation (CV) values for the different communities in the analysis. Figure A shows the $S A$ distributions for the different scenarios. The figure shows SADs for the highest relevant edge-removal threshold: thresholds just lower than threshold resulting in networks decomposition for S1, S2, S4 and S3 high threshold, and highest threshold before three communities are detected for S3 low thresholds.

Table A: SAD dispersion (CV) for detected communities in simulated scenarios

\begin{tabular}{ccllll}
\hline Scenario & Gene flow & P1 & P2 & P1P2 & P3 \\
\hline S1 & None & 0.0057 & 0.0048 & - & 0.0058 \\
S2 & Low & 0.0059 & 0.0051 & - & 0.0064 \\
S3 & Medium & $0.17 \dagger$ & $0.18 \dagger$ & - & $0.0059 \dagger$ \\
& & - & - & $0.064 \ddagger$ & $0.028 \ddagger$ \\
S4 & High & - & - & 0.0057 & 0.0065 \\
\hline
\end{tabular}

$\dagger$ High edge-removal thresholds

$\ddagger$ Low edge-removal thresholds 

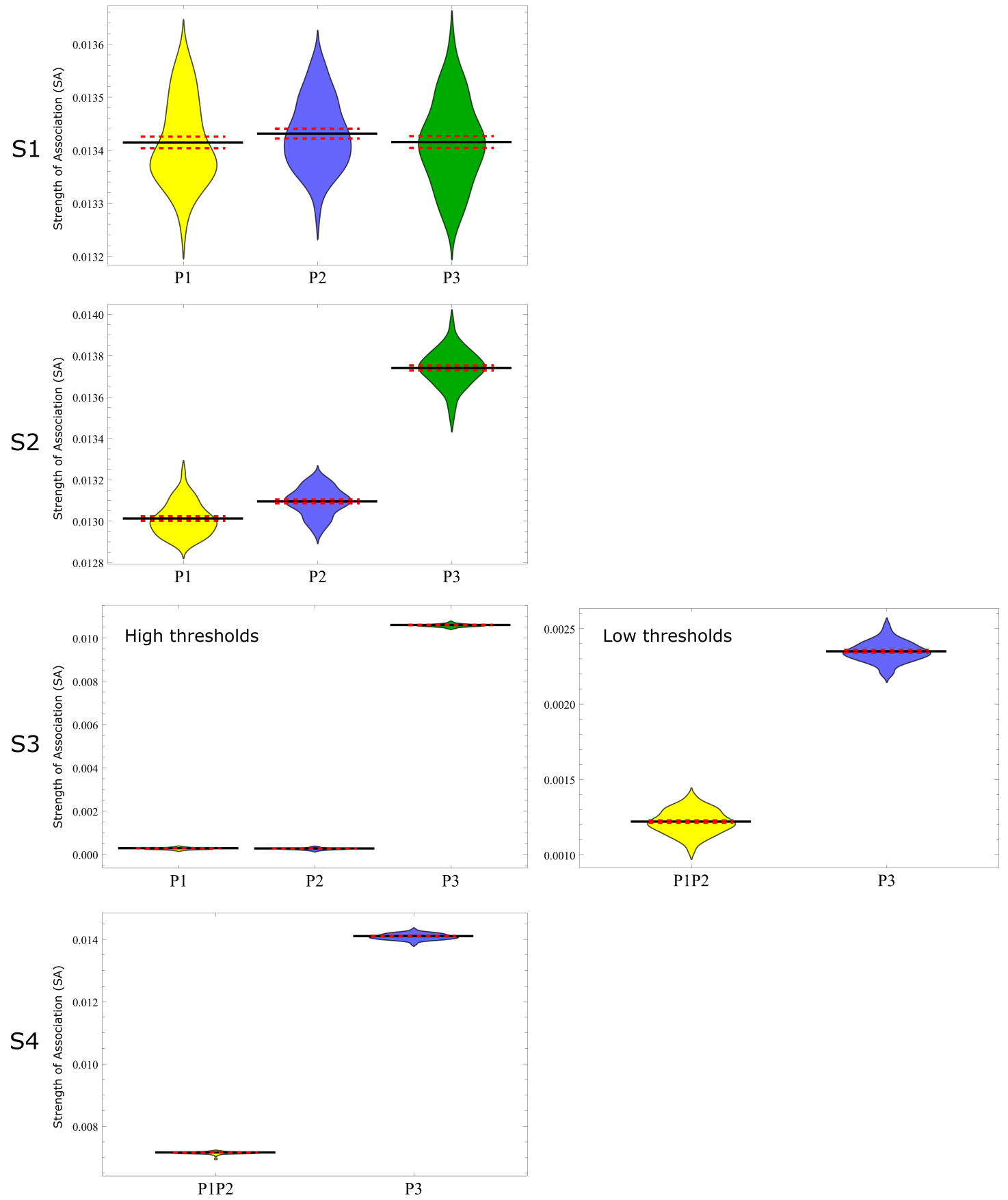

Figure A: SAD analysis of simulated scenarios. SAD means indicated by black line, Standard Errors indicated by dashed red lines. 


\section{Identifying hybrid individuals using SAD analysis}

Identification of hybrids was done by looking for outliers in the SAD for the different communities. $\mathrm{H} 1$ and H2 hybrid were simulated, with 20 repeats. For S1 and S2, both H1 and H2 were easily identified as outliers with noticeably lower SA values than the rest of the individuals in their assigned community. For S3, H1 was revealed as a hybrid with lower SA values for all simulations, but $\mathrm{H} 2$ was only clearly seen as an outlier for 6 out of the 20 repeated simulations. We therefore regarded $\mathrm{H} 2$ as undetectable in $\mathrm{S} 3$.

In order to try and determine the origin population of $\mathrm{H} 1$ and $\mathrm{H} 2$ hybrids, other than the subpopulation they were assigned to, the terms in equation 2 (in the main text) are examined. We considered the alternate subpopulation identifiable only if the corresponding $k$ resulted in a term lower than $90 \%$ of all other terms in equation 2 . Table B details the $\left(Q_{C}-Q_{C_{k}(i)}\right)$ terms of equation 2 for different $k$, and shows when and which subpopulation was identified as the origin of the hybrid. These results correspond to the example shown in Figure 6 in the main text, but the results were qualitatively identical for all 20 simulation repeats. For S1, the second community was identified only for H1, and it was identified correctly (as the non-assigned community of the pair $\mathrm{P} 1$ and $\mathrm{P} 2$ ). For S2, this analysis identified the correct population of origin for both $\mathrm{H} 1$ and $\mathrm{H} 2$ hybrids in all repeated simulations. For S3, the origin of H1 was identified correctly, and so was the origin of $\mathrm{H} 2$ when it was identified as an outlier.

Table B: Terms in equation $2\left(Q_{C}-Q_{C_{k}(i)}\right)$ for different $k$. Values identifying alternate subpopulation of origin of the hybrid individual ( $<90 \%$ of values for other terms) appear in bold. These results correspond with the simulation shown in Figure 6 in the main text, but results were qualitatively similar for all repeated simulations.

\begin{tabular}{cccccc}
\hline Scenario & Gene flow & Hybrid & P1 & P2 & P3 \\
\hline S1 & None & H1 & $0^{*}$ & $\mathbf{0 . 0 0 0 1 1}$ & 0.011 \\
& & H2 & $0^{*}$ & 0.012 & 0.012 \\
S2 & Low & H1 & $0^{*}$ & $\mathbf{0 . 0 0 0 0 7 7}$ & 0.012 \\
& & H2 & $0^{*}$ & $\mathbf{0 . 0 0 7 2}$ & 0.013 \\
S3† & \multirow{2}{*}{ Medium } & H1 & $0^{*}$ & $\mathbf{0 . 0 0 0 1 5}$ & 0.0053 \\
& & H2** & - & - & - \\
\hline
\end{tabular}

* Assigned community

** Not identified as outlier

$\dagger$ High edge-removal thresholds 